\title{
Correction of wandering smoothing effects on static measurements of a wing-tip vortex
}

\author{
Giacomo Valerio Iungo · Peter Skinner • \\ Guido Buresti
}

Received: 8 May 2008/Revised: 4 August 2008/Accepted: 2 September 2008/Published online: 9 October 2008

(C) Springer-Verlag 2008

\begin{abstract}
Wandering is a typical feature of wing-tip vortices and it consists in random fluctuations of the vortex core. Consequently, vortices measured by static measuring techniques appear to be more diffuse than in reality, so that a correction method is needed. In the present paper statistical simulations of the wandering of a Lamb-Oseen vortex are first performed by representing the vortex core locations through bi-variate normal probability density functions. It is found that wandering amplitudes smaller than $60 \%$ of the core radius are well predicted by using the ratio between the RMS value of the mean cross-velocity and its slope measured at the mean vortex center. Furthermore, the principal axes of wandering can be accurately evaluated from the opposite of the cross-correlation coefficient between the spanwise and the normal velocities measured at the mean vortex center. The correction of the wandering smoothing effects is then carried out through four different algorithms that perform the deconvolution of the mean velocity field with the probability density function that represents the wandering. The corrections performed are very accurate for the simulations with wandering amplitudes smaller than $60 \%$ of the core radius, whereas errors become larger with increasing wandering amplitudes. Subsequently, the whole procedure to evaluate wandering and to correct the mean velocity
\end{abstract}

G. V. Iungo $(\bowtie) \cdot$ G. Buresti

Department of Aerospace Engineering, University of Pisa, via Caruso, 56126 Pisa, Italy

e-mail: giacomo.iungo@ing.unipi.it

G. Buresti

e-mail: g.buresti@ing.unipi.it

P. Skinner

DPSS, CSIR, Pretoria, Republic of South Africa

e-mail: PSkinner@csir.co.za field is applied to static measurements, carried out with a fast-response five-hole pressure probe, of a tip vortex generated from a NACA 0012 half-wing model. It is found that the wandering is predominantly in the upward-outboard to downward-inboard direction. Furthermore, the wandering amplitude grows with increasing streamwise distance from the wing, whereas it decreases with increasing angle of attack and free-stream velocity.

\section{Introduction}

Tip vortices released from a large aircraft represent a significant hazard for other aircrafts that follow in its wake. This phenomenon affects the separation distance between aircrafts and, consequently, it remains a limiting factor for airport operation. Furthermore, the flow close to the wingtip is significant for a proper evaluation of the aerodynamic loads, of the flight mechanics characteristics and of the induced drag. In addition, a correct assessment of the velocity field of tip-vortices is fundamental in the design of ogee tips and winglets.

Wandering is a typical feature of wing-tip vortices and it consists in abrupt displacements of the vortex core location. In general, the objective of a wind-tunnel measurement of a wing-tip vortex is to characterize the vortex size and intensity, which would require performing measurements in a frame of reference that moves with the wandering vortex. However, when static (i.e., fixed-point) measurements are carried out, any time-averaged velocity value is actually a weighted average in both time and space. Consequently, the result is a smoothed vortex, with larger diameter and lower maximum tangential velocity than the real one, as if it were more diffuse. 
Chigier and Corsiglia (1972) and Corsiglia et al. (1973) compared measurements carried out by a fixed three-sensor hot-wire anemometer with tests performed using a rapid scanning technique, which consists in traversing an anemometer fixed on a rotating arm through the vortex core, to enable the latter to be considered roughly fixed during each scan. They found that static measurements are very susceptible to wandering. Fluctuations of the axial velocity signals were already observed by Green and Acosta (1991). They found oscillation amplitudes of the axial velocity as large as the free-stream velocity at the vortex centreline, and these fluctuations fell rapidly with increasing distance from the centreline. For an angle of attack of $10^{\circ}$ the fluctuations consisted of both "fast" and "slow" components, for $5^{\circ}$ only of the "fast" ones. The unsteadiness in tangential velocity was less than for the axial component, and it became larger by moving downstream. Shekarriz et al. (1992) observed from LDV measurements that the vortex seems to fluctuate primarily in the spanwise direction and less in the normal one. Also Yeung and Lee (1999) evaluated wandering characteristics using PIV data; they concluded that the wandering amplitude was comparable with the core radius and the maximum rate of wandering was roughly $4 \%$ of the freestream velocity. Regarding delta wings, Gursul and Xie (2000) attributed the random displacements of the vortex to the non-linear interaction of several small-scale vortices, generated by the Kelvin-Helmotz instability, with the primary vortex core.

Jaquin et al. (2001) proposed four possible causes for wandering: the vortex could be un-stabilized by wind-tunnel free-stream unsteadiness, turbulence in the surrounding shear layer, co-operative instabilities or propagation of unsteadiness from the model. They showed that wandering was apparently insensitive to the free-stream unsteadiness.

Surely, the strong point of the survey on wing-tip vortex wandering is the work of Devenport et al. (1996). The authors described wandering motion through a bi-variate normal probability density function, even though they did not support this assumption with any experimental data. The vortex is assumed to be axisymmetric, the wandering independent of any turbulent motion, and the velocities associated with the wandering itself negligible in comparison with those generated by the vortex. Obviously, all these hypotheses are not generally confirmed except for particular circumstances. With these assumptions, the mean velocity components and the mean Reynolds stresses, which correspond to the experimental data measured with static techniques, were expressed as the convolution of the actual field of those quantities with the bi-variate normal probability density function that represents the wandering. Furthermore, to solve the convolution integrals analytically, the axial velocity and the axial vorticity fields were fitted by sums of gaussian functions; this is not always experimentally possible (e.g., due to the presence of secondary vorticity structures) and, in addition, it is known that the tangential velocity profile of a fully rolled-up vortex is better represented by different models as, e.g., the Hoffmann and Joubert (1963) model. In summary, the fitting of the measured velocity field by gaussian functions may be a non-negligible error source, as possible flow asymmetries are not taken into account.

The bi-variate normal probability density function, which represents wandering, is characterized by two wandering amplitudes $\left(\sigma_{y}\right.$ and $\sigma_{z}$, for the spanwise and normal directions, respectively), and an anisotropy parameter, $e$. The latter represents the orientation of the principal axes of the vortex wandering with respect to the frame of reference. $\sigma_{y}$ and $\sigma_{z}$ are evaluated iteratively by dividing the root mean square value of the normal and spanwise velocities, respectively, with the tangential velocity gradient measured at the mean vortex center. Obviously, these quantities are a good index of the wandering amplitudes in both directions, but the authors did not support their procedure with any explanation or statistical simulations. Furthermore, the anisotropy parameter, $e$, is evaluated through the cross-correlation coefficient between the spanwise and normal velocities, measured at the mean vortex center. However, the authors did not explain how this quantity could be correlated with the directions of the principal axes of the vortex wandering and how the latter were evaluated. The preferred direction of wandering was observed by Devenport et al. (1996) to be between $53^{\circ}$ and $69^{\circ}$ in all cases, measured from the normal to the spanwise direction. The wandering amplitude was found to increase roughly as the square root of the downstream distance: from $10 \%$ of the core radius up to $35 \%$ moving downstream from 5 to 35 chordlengths. Wandering was responsible for $12 \%$ and $15 \%$ errors in the measured core radius and peak tangential velocity, respectively. The wandering amplitudes grew with increasing free-stream velocity, probably due to the increased wake turbulence, but they decreased with growing angle of attack. Devenport et al. (1996) concluded that the most important source of wandering is wind-tunnel unsteadiness and that, consequently, wandering decreases as the strength of the vortex is increased.

Conversely, Rokhsaz et al. (2000) showed that wandering amplitudes grow with increasing angle of attack, which is opposite to the finding of Devenport et al. (1996). Furthermore, the flow separation occurring at the higher angles of attack contributed to an increase in wandering.

Heyes et al. (2004) evaluated wandering effects by recentering PIV data. They assessed that the Devenport et al. 
(1996) assumption of using a bi-variate normal probability density function could be valid, and their corrections were in good agreement with those predicted by the Devenport et al. (1996) method. They found a $12.5 \%$ over-prediction of the core radius and a $6 \%$ under-prediction of the peak tangential velocity. The errors were larger for lower angles of attack. They also found that the wandering amplitude increases linearly with streamwise distance; a linear reduction was found by increasing the angle of attack, so that they concluded that the mechanism responsible for wandering is not self-induced, as had been proposed by Rokhsaz et al. (2000), but rather that the vortex is responding to an external perturbation, as for instance the background turbulence level, to which the tip vortex becomes less susceptible as the vortex strength is increased. For a more detailed bibliography regarding wing-tip vortices see Iungo and Skinner (2007).

In the present work static measurements of a vortex generated from a NACA 0012 half-wing model are carried out through a fast-response five-hole pressure probe. These tests highlight that while the turbulence coming from the wake vanishes traveling downstream due to viscosity, flow unsteadiness still persists in correspondence to the vortex core. By high-pass filtering the velocity signals, it is assessed that these persisting velocity fluctuations are characterized by very low non-dimensional frequencies (i.e., $f c / U_{\infty}<2$, where $f$ is frequency, $c$ is the mean geometric chord and $U_{\infty}$ is the free-stream velocity), and may thus be ascribed to vortex wandering.

Subsequently, the possibility of characterizing wandering from static measurements is analysed, and methods to correct the mean velocity field and the Reynolds stresses for wandering smoothing effects are investigated. First, the wandering of a Lamb-Oseen vortex is statistically simulated, for both 1D and 2D conditions. In order to represent the wandering motion, the vortex center is moved in subsequent time steps to locations derived from a bi-variate normal probability density function. For each analysed condition 1,500 vortex center positions, generated with the statistic software $R$, are simulated.

The statistical simulations confirm that the mean velocity field, affected by wandering smoothing effects, is essentially equal to the convolution of the actual velocity field of the Lamb-Oseen vortex with the bi-variate normal probability density function that represents the vortex wandering. Consequently, the correction of wandering smoothing effects on the mean velocity field consists in the deconvolution of the latter with the probability density function describing the wandering motion. The correction is performed using four different algorithms: the Van Cittert algorithm, the Richardson-Lucy algorithm, the blind deconvolution and a numerical direct deconvolution in the Fourier domain.
The advantage of all these methods consists in avoiding any hypothesis on the axisymmetry and/or on the shape of the flow field; consequently, errors due to the fitting of the measured data are avoided.

The paper is organized as follows. The five-hole probe static measurements are described in Sect. 2. The statistical simulations of the wandering of a Lamb-Oseen vortex are presented in Sect. 3 and the assessment of the methods to correct wandering effects on static measurements, using the simulated data, is reported in Sect. 4. The application of the whole procedure to characterize wandering from the five-hole probe static measurements and to correct the mean flow field for wandering effects is then described in Sect. 5. Finally, conclusions and recommendations are provided in Sect. 6.

\section{Five-hole probe static measurements}

\subsection{Experimental set-up and procedures}

The tests were performed in the Two Meter Wind Tunnel at the DPSS operating unit of the CSIR in Pretoria, South Africa. This facility is an open-circuit, open test-section, low-speed wind tunnel with a test-section diameter of $1.7 \mathrm{~m}$, a length of $2.55 \mathrm{~m}$ and a speed range between 3 and $33 \mathrm{~m} / \mathrm{s}$. For velocities higher than $5 \mathrm{~m} / \mathrm{s}$, the free-stream turbulence level is lower than $0.75 \%$, and a small negative axial pressure gradient is present $\left(\mathrm{d} C_{P} / \mathrm{d} x=-0.9 \% \mathrm{~m}^{-1}\right)$.

The tested model was a zero-sweep, untwisted halfwing, with NACA 0012 cross-sections, a blunt tip, aspect ratio of 5.7 and taper ratio of 0.4 . The wing semi-span was $0.7 \mathrm{~m}$ and the mean geometric chord, $c$, was $0.245 \mathrm{~m}$. In all tests no boundary layer trip was used on the model. The model was mounted in a vertical position on a base plate in such a way that the wing-tip was at a vertical distance of $0.15 \mathrm{~m}$ from the wind tunnel axis. This set-up ensured that for all the analysed stream-wise positions the tip vortex was sufficiently far from the free shear layer bounding the open test section. A mechanical apparatus was used to vary the wing angle of attack. The origin of the reference frame was located at the wing-tip trailing edge, with the $x$-axis in the free-stream direction and the $y$-axis in the spanwise direction, positive from root to tip; the $z$ direction was consequently defined, producing a clockwise frame of reference (see Fig. 1).

Static measurements were performed with an Aeroprobe fast-response five-hole pressure probe (5HP in the following), characterized by an external diameter of $1.59 \mathrm{~mm}$; the pressure transducers were connected to each orifice with $30 \mathrm{~mm}$ length tubes. The 5HP calibration was carried out between 5 and $35 \mathrm{~m} / \mathrm{s}$ in an appropriate facility of CSIR, which is a subsonic, low-turbulence wind tunnel, with open 


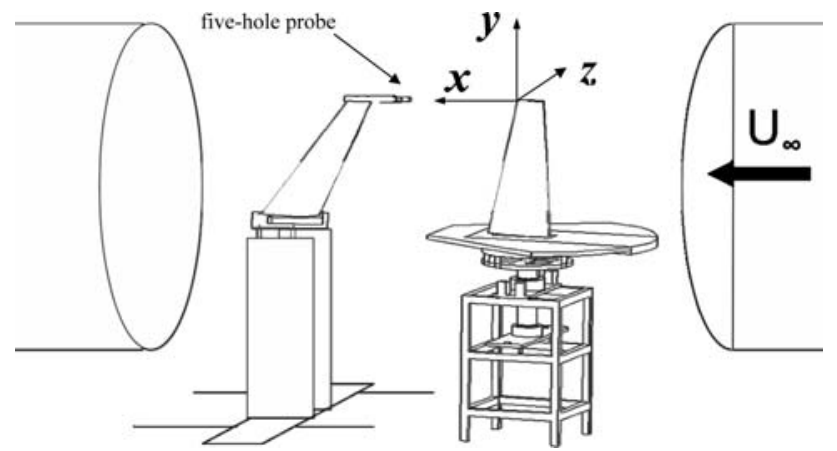

Fig. 1 Sketch of the experimental set-up

circuit and closed test section. The calibration method was derived from the one proposed for a seven-hole probe by Gerner and Maurar (1981), and the whole procedure is described in detail in Iungo and Skinner (2007).

During the tests the probe was mounted on its holder, and the whole system was fixed to a vertical wing-shaped support, and positioned downstream of the model on an automated traversing apparatus; the general set-up of the experiments is sketched in Fig. 1. In order to analyse the overall wake cross-section, measurement grids were first performed by moving the 5HP in planes perpendicular to the direction of the free-stream. For these measurements the space-step between each measurement point was constant and it was set from $2 \mathrm{~mm}$ up to $6 \mathrm{~mm}$, depending on the stream-wise position. The data sampling rate was $1 \mathrm{kHz}$ and the total sampling time was $5 \mathrm{~s}$ for each measurement point. Furthermore, traverses through the vortex center, in both the spanwise and normal directions, were carried out in order to obtain more detailed velocity profiles. For these tests the space-step between consecutive points was set to $0.5 \mathrm{~mm}$ and the sampling rate was $2 \mathrm{kHz}$, with a total sampling time of $33 \mathrm{~s}$ for each measurement.
A longer sampling period was necessary to achieve an adequate statistic and spectral characterization of the wandering phenomenon from static measurements.

Three main test series were performed. The objective of the first one was to analyse the downstream evolution of the vortex; for these tests the wing angle of attack, $\alpha$, was set at $8^{\circ}$ and the free-stream velocity, $U_{\infty}$, at $10 \mathrm{~m} / \mathrm{s}$ (corresponding to a Reynolds number of $R e=U_{\infty}$ $c / v \cong 169,000)$, while the stream-wise positions were varied from $0.1 c$ up to $6 c$. The second test series was carried out in order to evaluate the effects of the variation of the angle of attack, with $U_{\infty}=10 \mathrm{~m} / \mathrm{s}, x / c=6$ and the angle of attack set at $4^{\circ}, 8^{\circ}$ and $12^{\circ}$. Finally, to evaluate the $R e$-dependence of the vortex, tests were performed by positioning the model at $\alpha=8^{\circ}, x / c=6$ and setting the free-stream velocity at 10,20 and $30 \mathrm{~m} / \mathrm{s}$.

\subsection{Flow field analysis}

A deep analysis of the mean flow field obtained from the 5HP static measurements is reported in Iungo and Skinner (2007) and is not repeated here for the sake of brevity. The vortex characteristics evaluated for all tested conditions and locations are reported in Table 1 . In this table, $V_{\theta 1}$ is the peak of the cross-velocity (i.e., the modulus of the velocity component lying on a plane orthogonal to the vortex axis, which is assumed to be coincident with the $x$ axis) and the distance between its location and the mean vortex center is the core radius, $r_{1}$. The circulation evaluated at $r_{1}$ is denoted by $\Gamma_{1}$, whereas the theoretical circulation at the wing-root is $\Gamma_{0}$; the latter was derived in a previous investigation by comparing, for each angle of attack, the span-wise variation of the lift coefficient obtained from pressure measurements with the results of a potential-flow code. The axial velocity deficit in

Table 1 Vortex parameters for the tested conditions and locations

\begin{tabular}{llllllllll}
\hline$N_{o}$ & $\alpha(\mathrm{deg})$ & $U_{\infty}(\mathrm{m} / \mathrm{s})$ & $x / c$ & $r_{1} / c$ & $V_{\theta 1} / U_{\infty}$ & $U_{D} / U_{\infty}$ & $\Gamma_{1} / \Gamma_{0}$ & $\Gamma_{1} / v$ & $U_{D} / V_{\theta 1}$ \\
\hline G 01 & 8 & 10 & 0.1 & 0.0294 & 0.580 & -0.373 & 0.346 & 18,100 & -0.643 \\
G 02 & 8 & 10 & 0.33 & 0.0337 & 0.576 & -0.221 & 0.393 & 20,600 & -0.384 \\
G 03 & 8 & 10 & 0.66 & 0.0408 & 0.569 & -0.205 & 0.471 & 24,600 & -0.360 \\
G 04 & 8 & 10 & 1 & 0.0354 & 0.395 & -0.119 & 0.283 & 14,800 & -0.301 \\
G 05 & 8 & 10 & 1.5 & 0.0374 & 0.356 & -0.124 & 0.270 & 14,200 & -0.348 \\
G 06 & 8 & 10 & 2 & 0.0335 & 0.346 & -0.046 & 0.235 & 12,300 & -0.132 \\
G 07 & 8 & 10 & 3 & 0.0426 & 0.284 & -0.101 & 0.245 & 12,800 & -0.356 \\
G 08 & 8 & 10 & 6 & 0.0543 & 0.182 & -0.098 & 0.200 & 10,500 & -0.541 \\
G 09 & 8 & 20 & 6 & 0.0410 & 0.316 & -0.075 & 0.264 & 27,700 & -0.236 \\
G 10 & 8 & 30 & 6 & 0.0314 & 0.358 & -0.041 & 0.228 & 35,800 & -0.113 \\
G 11 & 4 & 10 & 6 & 0.0723 & 0.098 & -0.098 & 0.287 & 7,550 & -0.997 \\
G 12 & 12 & 10 & 6 & 0.0488 & 0.297 & -0.015 & 0.207 & 15,400 \\
\hline
\end{tabular}


correspondence to the mean vortex center, $U_{D}$, is evaluated as the difference between the measured axial velocity and the free-stream velocity, $U_{\infty}$.

Analysing the tests performed at different stream-wise distances from the wing, a reduction of the peak crossvelocity, $V_{\theta 1}$, by moving downstream is apparent, which suggests that a diffusion process of the vortex is taking place due to viscosity. However, the core radius, $r_{1}$, grows at a much lower rate than predicted in Batchelor (1964), where it is assumed that a reduction of $V_{\theta 1}$, due to the vorticity diffusion, implies the same rate of increase of $r_{1}$ due to mass conservation. This would support the idea that this apparent diffusion of the vortex might be the result of a smoothing effect due to wandering or, at least, that the vortex diffusion is taking place at a much lower rate.

Subsequently, the unsteadiness of the velocity components was analysed in order to assess the presence of vortex wandering. In Fig. 2 the non-dimensional variance of the axial velocity, $\left(\sigma_{U} / U_{\infty}\right)^{2}$, is plotted for several locations tested with the measurement grids. It is evident that in the very near field the velocity fluctuations are high; at $x / c=3$, $\sigma_{U}$ is greatly decreased in the wake, whereas a significant unsteadiness is still present in correspondence to the vortex core up to $x / c=6$. Analogous results may be found for the variance of the cross-velocity, not reported here. It is then fundamental to understand whether this unsteadiness in correspondence to the vortex core is the result of a real turbulent activity or, as suggested by Bandyopadhyay et al. (1991), Devenport et al. (1996) and Chow et al. (1997), the vortex core is a relaminarization region, which far downstream is characterized by fluctuations at relatively small frequencies that may be ascribed to vortex wandering, i.e., to the oscillation of vorticity structures with dimensions comparable to the vortex core itself.
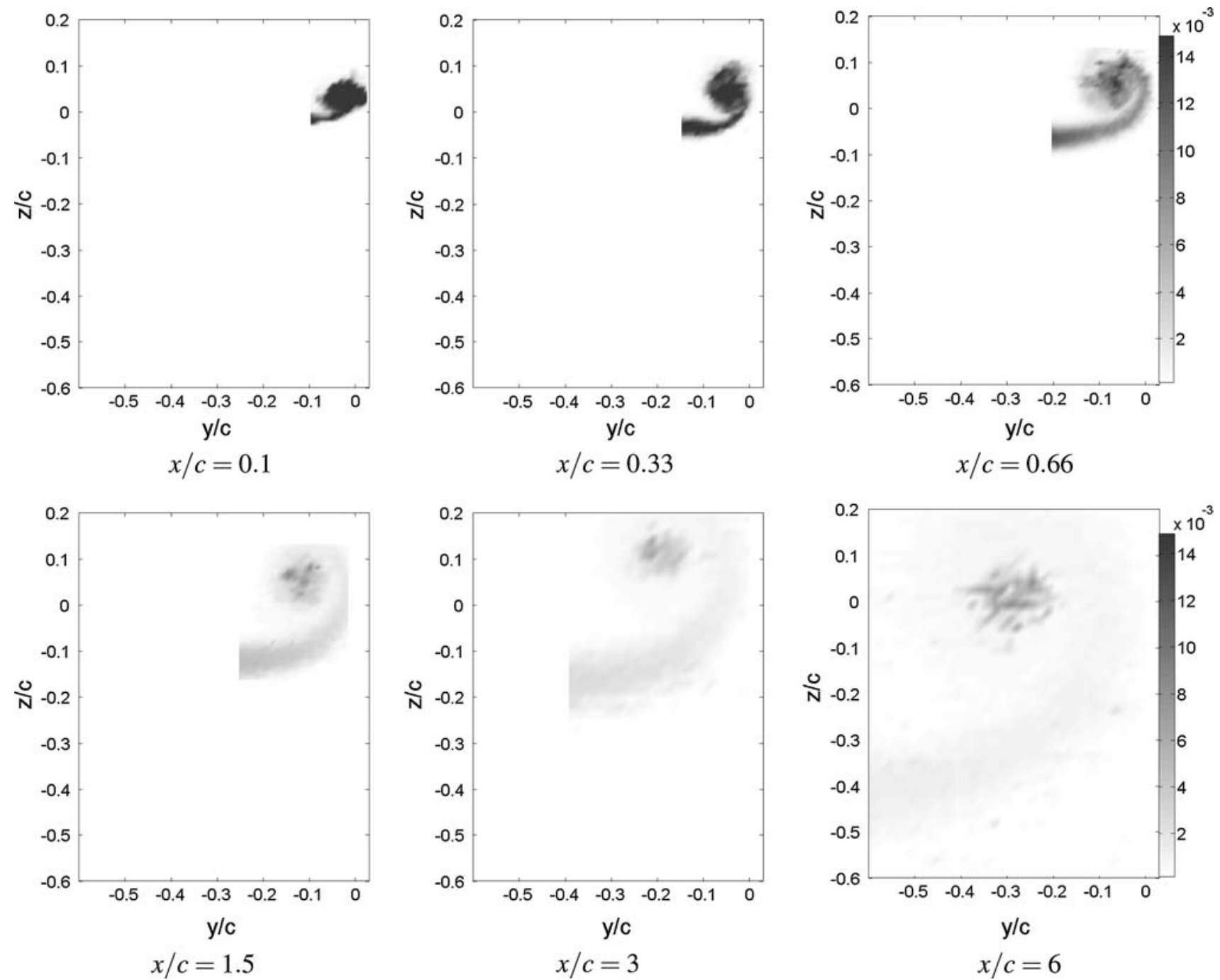

Fig. 2 Non-dimensional variance of the axial velocity, $\left(\sigma_{U} / U_{\infty}\right)^{2}$, for the condition $\alpha=8^{\circ}, U_{\infty}=10 \mathrm{~m} / \mathrm{s}$. All figures are plotted with the same colour scale (white $=0$ and black $=0.015$ ) 
From the spectral analysis of the velocity signals, no definite conclusion could be drawn; in effect, by approaching the vortex center along a radial direction, both the Fourier and wavelet velocity spectra showed an energy increase extended to the whole frequency domain; this behavior was especially enhanced for the cross-velocity. Furthermore, for a fixed radial distance from the vortex center, a similar increase of energy was observed by moving downstream.

In order to characterize the persistent fluctuations at the vortex core, the signals obtained from the static measurements were then filtered using high-pass filters with different cut-off frequencies. In analogy with the spectral analysis performed by Beninati and Marshall (2005), the chosen cut-off frequencies correspond to wavelengths that are multiples of the core radius, i.e., 200, 100, 30 and 10 times the core radius (the considered $r_{1}$ value is about $0.05 c$ ). Therefore, the used cut-off frequencies were $f_{\text {th } 1}=4 \mathrm{~Hz}, f_{\text {th } 2}=8 \mathrm{~Hz}, f_{\text {th } 3}=27 \mathrm{~Hz}$ and $f_{\text {th } 4}=81 \mathrm{~Hz}$; these values correspond, respectively, to non-dimensional frequencies $\left(f c / U_{\infty}\right)$ of $0.1,0.2,0.66$ and 1.98, and allowed the progressive disappearance of the wandering-related fluctuation energy to be highlighted.

As can be seen from Fig. 3, at the location $x / c=0.1$ the filtering does not produce any changes to the non-dimensional variance of both the axial and cross velocities. Small effects of the filtering are observed already at $x / c=0.66$, but the more interesting results for the location $x / c=6$ are
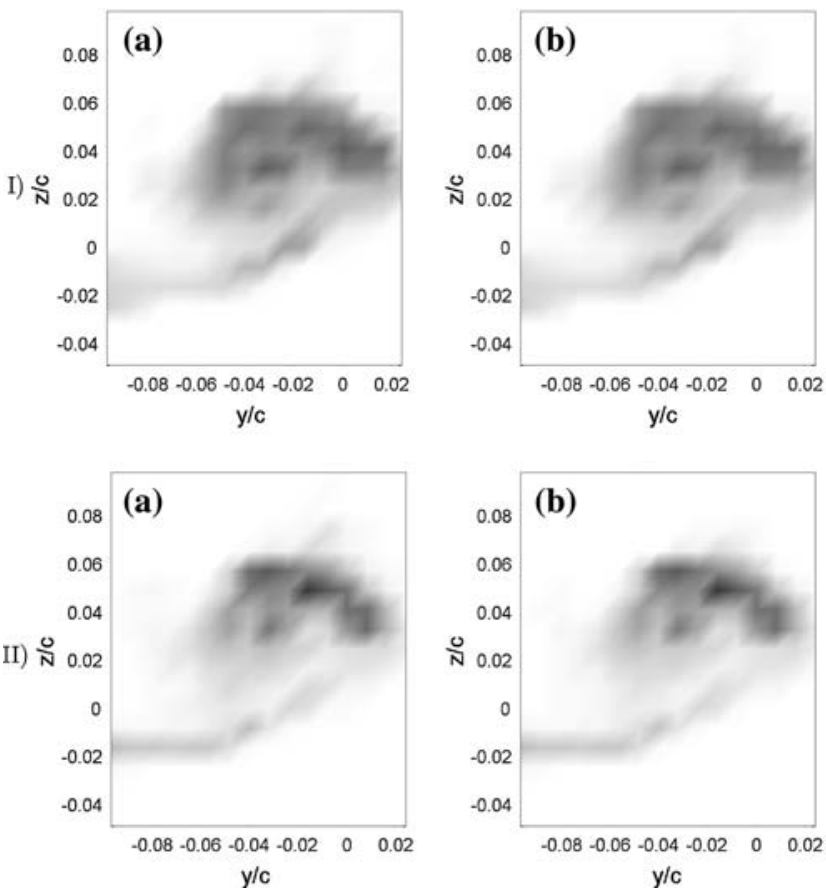

shown in Fig. 4. For both the axial and cross velocities it is seen that with increasing cut-off frequency the unsteadiness in the wake remains almost unchanged whereas in correspondence to the vortex core it disappears completely for the highest cut-off frequency. In other words, the fluctuations in the vortex core show a decreasing content at the higher frequencies by proceeding downstream, where the unsteadiness is mainly characterized by low frequencies, and cannot thus be considered the result of a real turbulent activity but rather of a low-frequency instability of the vortex, viz. the wandering. It is now fundamental to assess if the wandering characteristics may be derived from static measurements and to investigate the performance of methods to correct the data for wandering smoothing effects.

\section{Wandering statistical simulations}

A simulation of the wandering of a Lamb-Oseen vortex was carried out in order to assess the possibility of characterizing vortex wandering from static velocity measurements. The statistical simulations were carried out through the statistical software $R$, which is a language and environment for statistical computing and graphics. It can be accessed through the World Wide Web at http:// www.r-project.org. For our statistical simulations the generation of the vortex center locations was performed
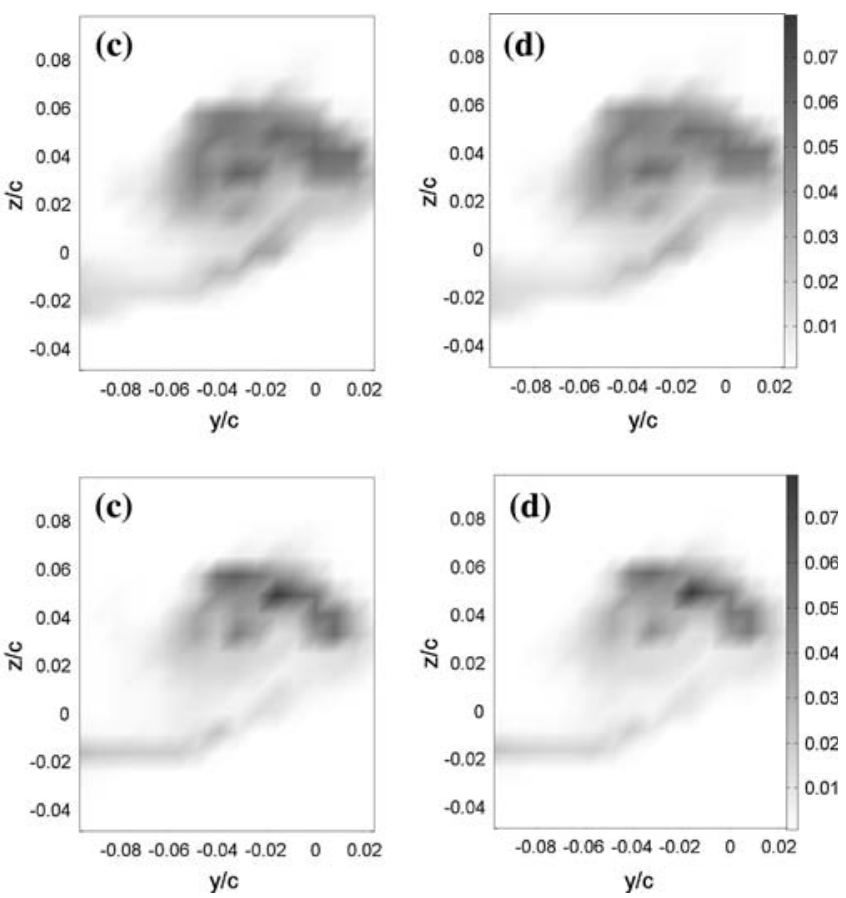

Fig. 3 Non-dimensional variance for the condition $x / c=0.1, \alpha=8^{\circ}, U_{\infty}=10 \mathrm{~m} / \mathrm{s}$ of the axial velocity (I) and of the cross-velocity (II), high-pass filtered with different cut-off frequencies: $\mathbf{a} f_{\mathrm{th} 1}=4 \mathrm{~Hz} ; \mathbf{b} f_{\mathrm{th} 2}=8 \mathrm{~Hz} ; \mathbf{c} f_{\mathrm{th} 3}=27 \mathrm{~Hz} ; \mathbf{d} f_{\text {th } 4}=81 \mathrm{~Hz}$. (Colour scale: white $=0$ and black $=0.08$ ) 

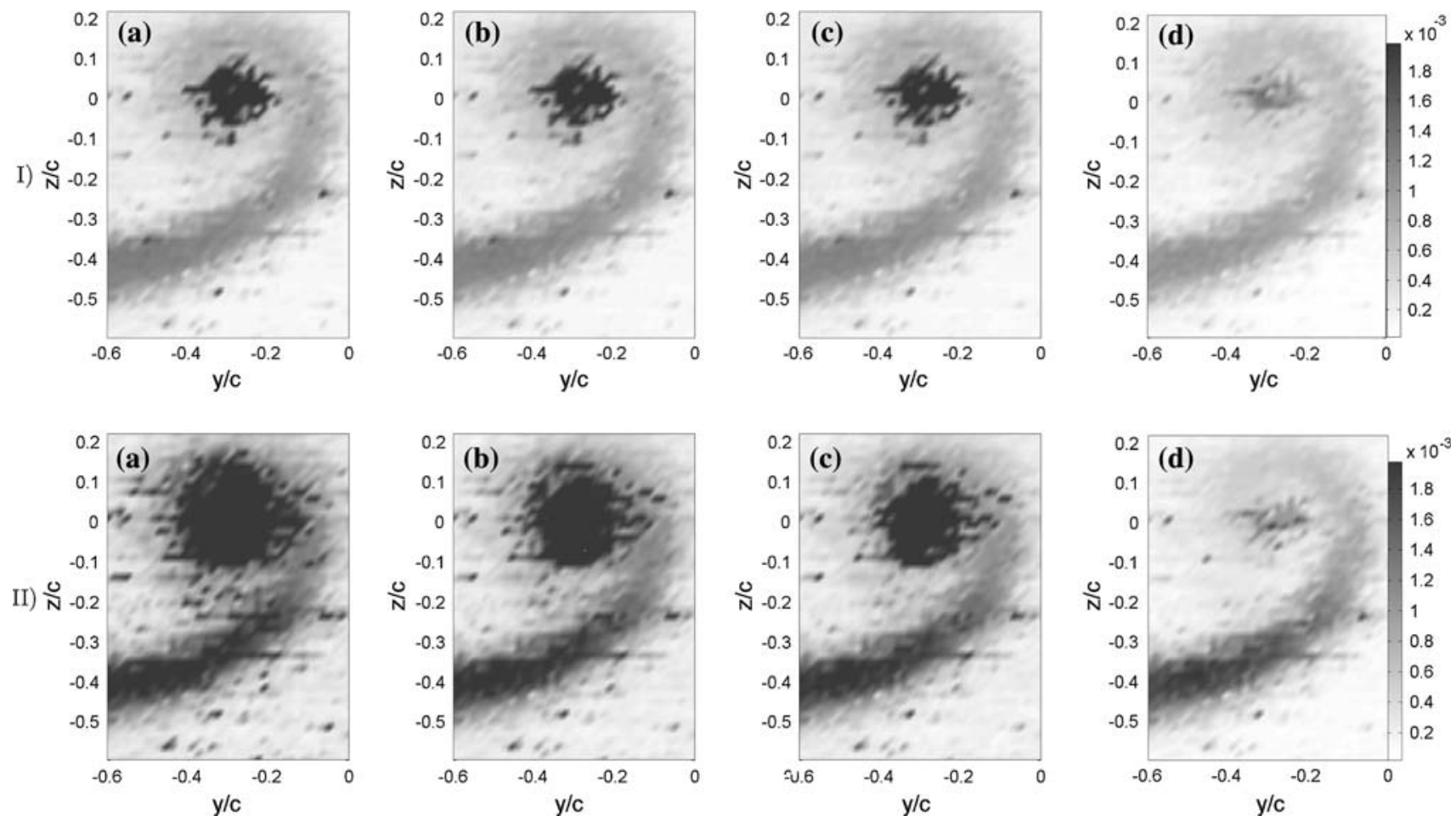

Fig. 4 Non-dimensional variance for the condition $x / c=6, \alpha=8^{\circ}, U_{\infty}=10 \mathrm{~m} / \mathrm{s}$ of the axial velocity (I) and of the cross-velocity (II), highpass filtered with different cut-off frequencies: $\mathbf{a} f_{\text {th } 1}=4 \mathrm{~Hz} ; \mathbf{b} f_{\text {th2 }}=8 \mathrm{~Hz} ; \mathbf{c} f_{\text {th } 3}=27 \mathrm{~Hz} ; \mathbf{d} f_{\text {th } 4}=81 \mathrm{~Hz}$. (Colour scale: white $=0$ and black $=0.002$ )

through the mvrnorm tool, which was implemented following Ripley (1987).

Two main test cases were used: the first with a peak cross-velocity $V_{\theta 1}=0.4$ and a core radius $r_{1}=1$, the second with $V_{\theta 1}=0.7$ and $r_{1}=1$. The vortex wandering was simulated by representing the vortex center locations through a bi-variate normal probability density function $(2 V d F)$, as proposed by Devenport et al. (1996) and confirmed by Heyes et al. (2003). A $2 V d F$ is described by the following equation:

$$
\begin{aligned}
p(y, z)= & \frac{1}{2 \pi \sigma_{y} \sigma_{z} \sqrt{1-e^{2}}} \\
& \times \exp \left[-\frac{1}{2\left(1-e^{2}\right)}\left(\frac{y^{2}}{\sigma_{y}^{2}}+\frac{z^{2}}{\sigma_{z}^{2}}-\frac{2 e y z}{\sigma_{y} \sigma_{z}}\right)\right]
\end{aligned}
$$

where $\sigma_{y}$ and $\sigma_{z}$ represent the wandering amplitudes along the $y$ - and $z$-axes, respectively, and $e$ is the term that represents the anisotropy of the motion with respect to the adopted frame of reference.

Once the wandering amplitudes, $\sigma_{y}$ and $\sigma_{z}$, and the anisotropy parameter, $e$, were chosen, each simulation was based on the generation, through the statistical software $R$, of 1,500 points described by a probability density function equal to the chosen $2 V d F$. Each point represents the location of the vortex center for each time-step of the simulation. In Fig. 5 the vortex centers generated for three different $2 V d F$ are shown.

The wandering amplitudes investigated were from 0.1 up to 1.2 times the core radius, and the parameter $e$ was set from -1 up to 1 with increments of 0.1 . Many combinations of these three parameters were simulated.

Once the position of the vortex center for each snapshot was known, the cross-velocity field generated by a LambOseen vortex could be evaluated. Consequently, for each point of the space domain a velocity signal was generated which was analogous to one that might have been obtained from a static velocity measurement. In the following the statistical simulations of the wandering of a Lamb-Oseen vortex with $V_{\theta 1}=0.4$ and $r_{1}=1$ are described, and the corresponding actual cross-velocity field of the vortex is shown in Fig. 6a. All the following figures of the velocity field, regarding the present condition, are plotted with a grey scale where the colour white indicates the value 0 and the black the peak cross-velocity value $V_{\theta 1}=0.4$. For the present simulation the wandering is simulated with $\sigma_{y} / r_{1}=0.4, \sigma_{z} / r_{1}=0.3$ and $e=0.2$. The statistical simulation confirms that the mean cross-velocity field (Fig. 6b) is well represented by the convolution of the actual crossvelocity field with the $2 V d F$ (Fig. 6c). Some differences are observed only in proximity of the boundary of the space domain due to the finiteness of the latter. 

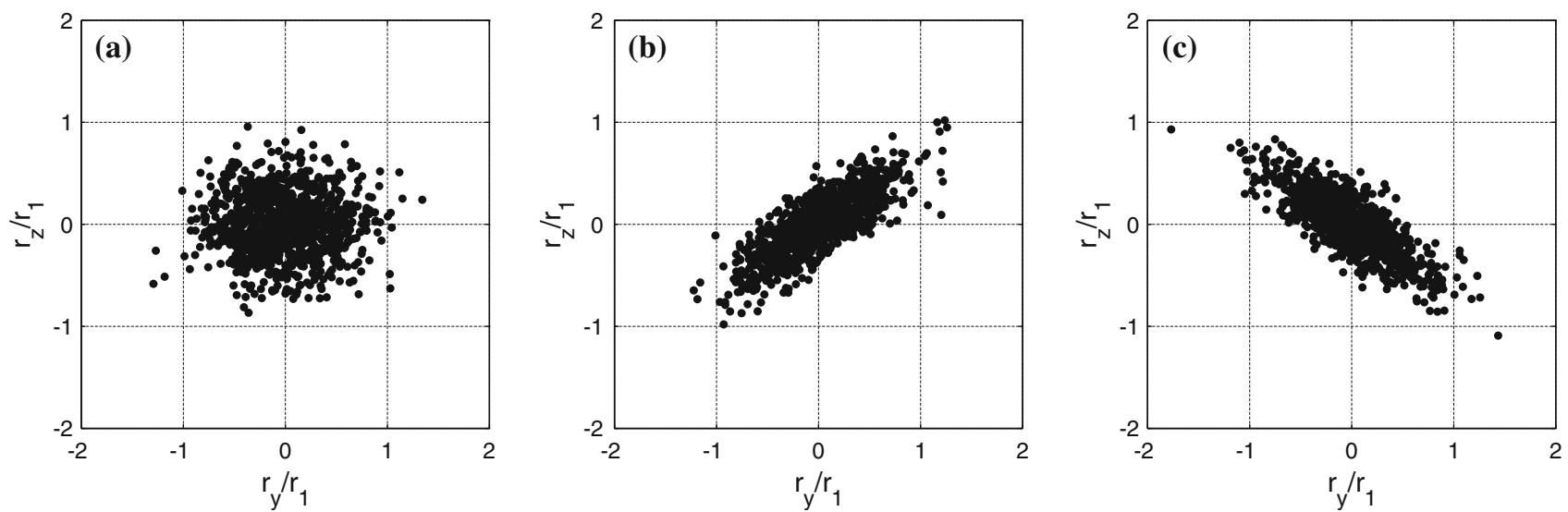

Fig. 5 Vortex center locations simulated with the statistical software $R$ for different probability density functions, $2 V d F$ : a $\sigma_{y} / r_{1}=0.4$, $\sigma_{z} / r_{1}=0.3, e=0 ; \mathbf{b} \sigma_{y} / r_{1}=0.4, \sigma_{z} / r_{1}=0.3, e=0.8 ; \mathbf{c} \sigma_{y} / r_{1}=0.4, \sigma_{z} / r_{1}=0.3, e=-0.8$
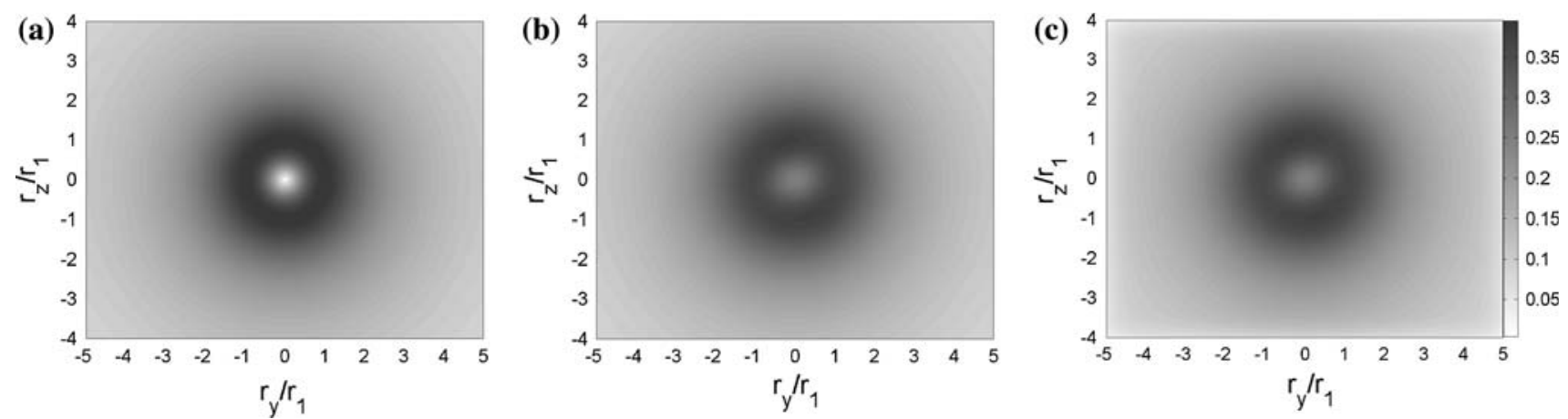

Fig. 6 Statistical simulation of the wandering of a Lamb-Oseen vortex with $V_{\theta 1}=0.4$ and $r_{1}=1$. Wandering is simulated with $\sigma_{y} / r_{1}=0.4$, $\sigma_{z} / r_{1}=0.3, e=0.2$ : a actual cross-velocity field; $\mathbf{b}$ mean cross-velocity field; $\mathbf{c}$ convolution of the actual cross-velocity field with the $2 V d F$ that represents the wandering. (Colour scale: white $=0$ and black $=0.4$ )

Devenport et al. (1996) proposed to evaluate the wandering amplitude $\sigma_{y}\left(\sigma_{z}\right)$ as the ratio of the RMS and the gradient of the normal velocity $W$ (spanwise velocity $V$ ), measured at the mean vortex center, possibly low-pass filtering the velocity signals in order to separate the fluctuations due to wandering from the fluctuations generated by turbulence. This criterion was not supported by any explanation or data.

The wandering amplitude $\sigma_{y}$ predicted from the statistical simulations using the above-mentioned method is plotted as a function of the actual simulated wandering amplitude in Fig. 7. Each value of the predicted wandering amplitude $\sigma_{y}$ is calculated as the average of at least three different values of the normal wandering amplitude, $\sigma_{z}$, and three anisotropy parameter values. As is apparent, the wandering amplitudes are accurately evaluated for actual wandering amplitudes smaller than $60 \%$ of the core radius. For higher values the error increases with increasing simulated wandering amplitude, reaching errors of $35 \%$ of the actual value for wandering amplitudes comparable to the core radius. Several simulations were also performed with 3,000 snapshots, i.e., twice longer than the usual

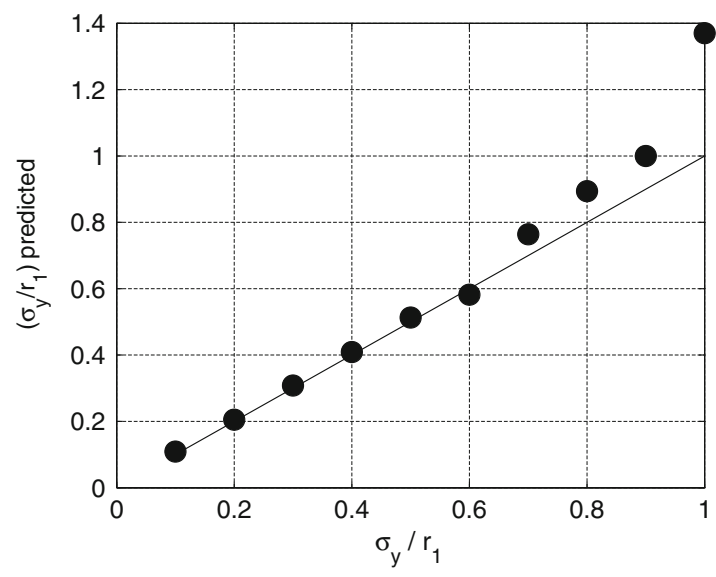

Fig. 7 Predicted wandering amplitude as a function of the actual wandering amplitude obtained from the statistical simulations

simulations, but the errors on the evaluation of the wandering amplitudes remained unaltered. Therefore, from our statistical simulations the method proposed by Devenport et al. (1996) is confirmed to be the best way to determine the wandering amplitudes from static measurements. 
The parameter $e$ of the $2 V d F$ represents the anisotropy of the vortex wandering with respect to the adopted frame of reference. It was calculated by Devenport et al. (1996) from the cross-correlation coefficient between the spanwise $V$ and the normal $W$ velocities, $\overline{V W} / \sigma_{V} \sigma_{W}$, measured at the mean vortex center. Furthermore, for each cross-section of the wake these authors found a direction with prevalently negative values of $\overline{V W} / \sigma_{V} \sigma_{W}$.

From the present experimental campaign it is confirmed that for all tested conditions and locations a particular direction with negative values of the cross-correlation coefficient between the spanwise and normal velocities, $\overline{V W} / \sigma_{V} \sigma_{W}$, is generally found, as shown in Fig. 8a for a typical condition. Furthermore, from Fig. $8 \mathrm{~b}$ it is interesting to observe that in correspondence to the mean vortex center location this anisotropy vanishes by high-pass filtering the velocity signals; in fact, for the highest cut-off frequency $\overline{V W} / \sigma_{V} \sigma_{W}$ becomes roughly zero at $r / c=0$. This indicates that the flow anisotropy is an effect only of wandering and not a general characteristic of all scales of the vorticity structures, at variance with the findings of Beninati and Marshall (2005).

From the statistical simulations, maps of $\overline{V W} / \sigma_{V} \sigma_{W}$ were then evaluated, like the ones relative to three different values of the anisotropy parameter, $e$, reported in Fig. 9. For the case with $e=0$ the cross-correlation coefficient $\overline{V W} / \sigma_{V} \sigma_{W}$ is roughly zero in correspondence to the mean vortex center, i.e., at $\left(r_{y} / r_{1}, r_{z} / r_{1}\right)=(0,0)$. When the case $e=1$ is simulated, a cross-correlation coefficient $\overline{V W} / \sigma_{V} \sigma_{W} \cong-1$ is found at the mean vortex center, whereas for $e=-1$ it is roughly equal to 1 . Therefore, the anisotropy parameter, $e$, of the $2 V d F$ can be predicted from the opposite of the value of the cross-correlation coefficient $\overline{V W} / \sigma_{V} \sigma_{W}$ evaluated in correspondence to the mean vortex center. This feature was not highlighted in Devenport et al. (1996) and Heyes et al. (2003).

This result may better be understood from the sketch reported in Fig. 10, in which the variations of the spanwise $V$ and normal $W$ velocities at the mean vortex center are evaluated when the vortex leaves this location. If the vortex moves along the $y$-axis (z-axis) only the velocity $W(V)$ varies, hence the cross-correlation between $V$ and $W$ is null. This situation corresponds to an isotropic wandering, hence $\overline{V W} / \sigma_{V} \sigma_{W}=0$ means $e=0$. If $e=1$ it means that the principal axes of wandering are rotated by $45^{\circ}$ with respect to the frame of reference. In this situation the variation of $V$ and $W$ have the same modulus and opposite sign, as
Fig. 8 Cross-correlation coefficients between the spanwise and the normal velocities, $\overline{v w} / \sigma_{V} \sigma_{W}$, for the condition $\alpha=8^{\circ}$,

$U_{\infty}=10 \mathrm{~m} / \mathrm{s}, x / c=6:$ a map obtained from the measurement grid (the black points represent the wake centreline);

b spanwise cross-section of $\overline{\nu w} / \sigma_{V} \sigma_{W}$ evaluated by highpass filtering the velocity signals with different cut-off frequencies
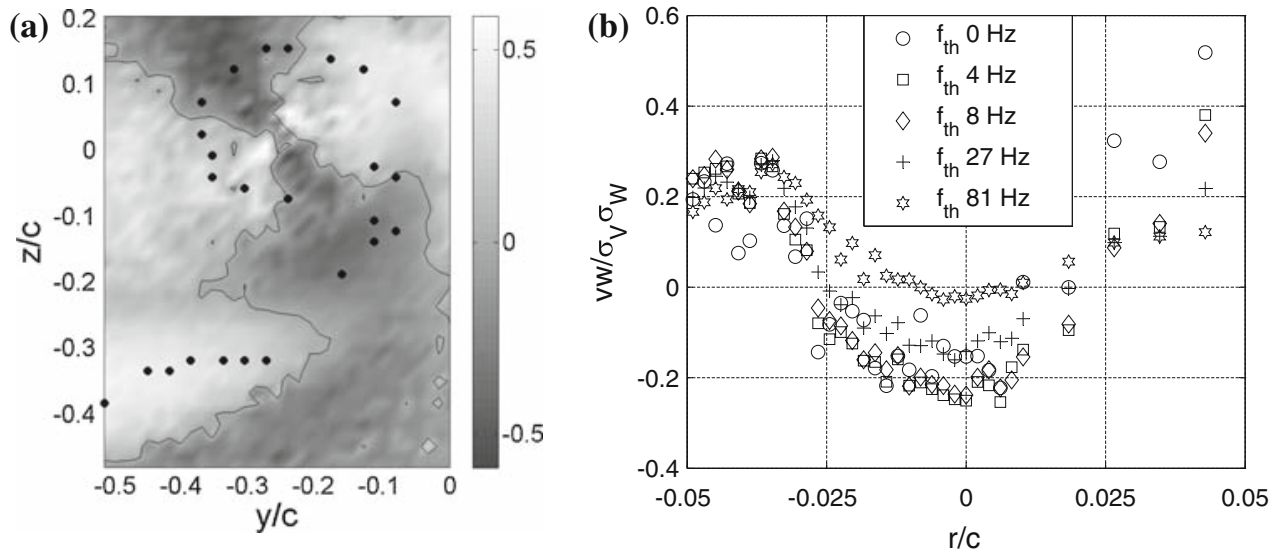
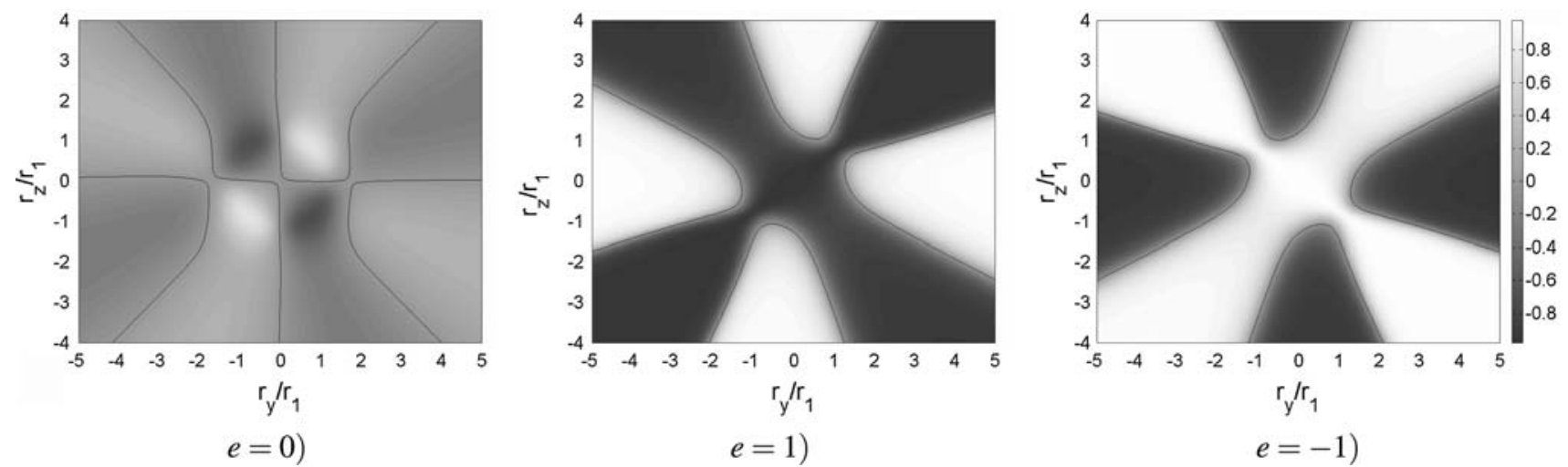

Fig. 9 Maps of the cross-correlation coefficient, $\overline{V W} / \sigma_{V} \sigma_{W}$, between the spanwise and normal velocities for different values of the anisotropy parameter, $e$, obtained from the statistical simulations. (Iso-contours relative to the value zero are reported with a solid line) 


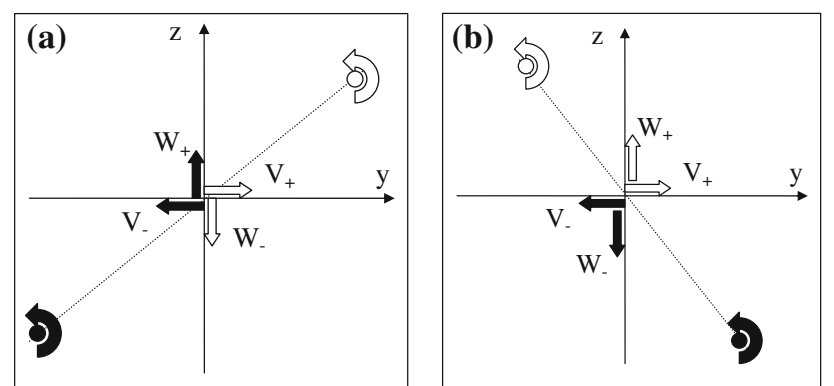

Fig. 10 Sketch of the contributions to the cross-correlation coefficient $\overline{V W} / \sigma_{V} \sigma_{W}$ at the mean vortex center as a function of the orientation of the principal axes of wandering: $\mathbf{a} e=1 ; \mathbf{b} e=-1$

reported in Fig. 10a, hence $\overline{V W} / \sigma_{V} \sigma_{W}=-1$. Analogously, when $e=-1$ the parameter $\overline{V W} / \sigma_{V} \sigma_{W}$ has the opposite value.

From the statistical simulations we found that the errors on the prediction of $e$ from the opposite of the value of $\overline{V W} / \sigma_{V} \sigma_{W}$, evaluated in correspondence to the mean vortex center, are negligible.

Summarizing, the statistical simulations of the wandering of a Lamb-Oseen vortex confirm that wandering can be characterized from static measurements. The wandering amplitudes are very accurately evaluated from the ratio of the RMS value of the cross-velocity and its gradient measured at the mean vortex center, as proposed by Devenport et al. (1996), for wandering amplitudes smaller than $60 \%$ of the core radius. The error increases with increasing wandering amplitudes, up to $35 \%$ of the actual value for wandering amplitudes comparable to the core radius. Furthermore, the parameter $e$, which represents the anisotropy of wandering, can be obtained from the opposite of the value of the cross-correlation coefficient between the spanwise $V$ and the normal $W$ velocities, $\overline{V W} / \sigma_{V} \sigma_{W}$, measured at the mean vortex center.

\section{Methods to correct wandering smoothing effects on static measurements}

As shown in the previous section, if wandering is assumed to be represented through a bi-variate normal probability density function, the main parameters of this function may be well evaluated from statistical quantities directly derived from the wandering-affected data. Now the problem of the correction of the results of static measurements to obtain the actual velocity field of the vortex will be considered.

The statistical simulations confirmed that the mean velocity field may be accurately predicted from the convolution of the actual velocity field of the vortex with the $2 V d F$ that characterizes the wandering. Consequently, the correction of the mean velocity field consists in the deconvolution of the latter with the $2 V d F$, as proposed by Devenport et al. (1996). These authors performed the deconvolution by solving the convolution integrals analytically. To this end, the velocity fields are supposed to be axisymmetric and the actual and the mean velocity fields are both expressed as sum of gaussian functions in order to solve the convolution integrals analytically. This procedure requires the fitting of the measured velocity fields with sum of gaussian functions; however, this may introduce an error that, in extreme circumstances, might be comparable to the performed correction. Furthermore, with this method the averaging effects due to possible secondary vorticity structures, which may surround the main vortex, or, more in general, the asymmetry of the flow field are not taken into account.

For this reason other deconvolution methods were investigated which do not need any assumption or preconditioning of the mean velocity field. Deconvolution techniques have been used in several applications, in particular in image and signal deblurring. In the present work four different procedures were used, in order to compare their performance. The measured velocity field $g(\mathbf{x})$, where $\mathbf{x}$ is the position vector, is expressed in the space domain as the convolution of the probability density function $\operatorname{pdf}(\mathbf{x})$, which describes the vortex wandering, with the actual velocity field $h(\mathbf{x})$ :

$g(\mathbf{x})=\operatorname{pdf}(\mathbf{x}) * h(\mathbf{x})$

where * represents the convolution operator. In the Fourier domain this application can be expressed as follows:

$G(\omega)=\operatorname{PDF}(\omega) H(\omega)$

The deconvolution is then mathematically computed from:

$H(\omega)=\frac{G(\omega)}{\operatorname{PDF}(\omega)}$

This application is ill-posed, and numerical problems arise because the probability density function and the measurements are both band-limited due to the finite size of the measurement domain. Appropriate numerical procedures must then be used to overcome this difficulty. The first method used in the present work is the Van Cittert algorithm, see Jansson (1984), which performs the deconvolution using the following approximation:

$h=\sum_{i=0}^{N}(I-\mathrm{pdf})^{i} * g$

where $I$ is the identity operator. In other words, the deconvolution is evaluated through the algebraic sum of the measured data and multiples of its recursive convolutions. For our application $N$ was set equal to 5 .

The second method is based on the Richardson-Lucy algorithm, see Richardson (1972). This technique consists 


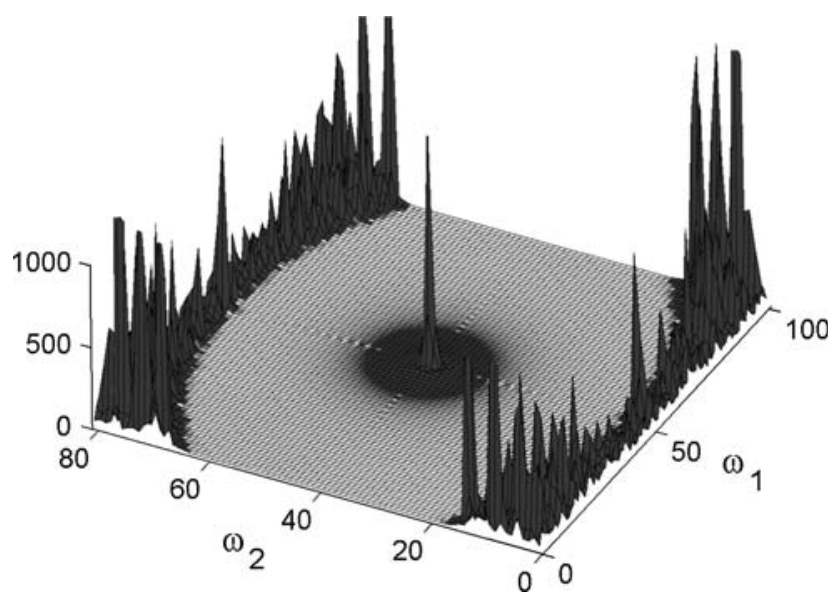

Fig. 11 Deconvoluted velocity field in the Fourier domain without any conditioning

in maximizing the likelihood of the deconvoluted velocity field. The third technique is a blind deconvolution, see e.g., Larson (2002): this technique is called blind deconvolution because the $2 V d F$, which represents the wandering, is unknown a priori and is iteratively estimated directly from the mean flow field. In other words, the mean flow field is corrected and, simultaneously, an optimization of a first estimation of the $2 V d F$ is performed. Both these two last algorithms are easily available from commercial mathematical softwares. For these methods it is fundamental to determine the number of iterations to be performed: with few iterations they produce a velocity field which is not strongly filtered (i.e., in the Fourier domain only the low spatial frequencies are well represented), whereas with many iterations noise amplification problems may arise.

The last method used in the present work was the direct calculation of the deconvolution in the Fourier domain. As already pointed out, the deconvolution is an ill-posed problem and in the Fourier domain it generates several spurious spectral contributions, which surround the contributions that represent the velocity field (Fig. 11). These spurious contributions are neglected, so that the inverse Fourier transform produces the actual velocity field. When the wandering amplitude is comparable with the core radius the spurious contributions may be very close to the physical contributions, and neglecting them may produce an inaccurate estimation of the actual velocity field.

In the present work the performance of the various procedures was assessed by using the statistical simulations already presented in Sect. 3. The simulation of the wandering of a Lamb-Oseen vortex with $V_{\theta 1}=0.4$ and $r_{1}=1$ is described, in which the wandering is first simulated with $\sigma_{y} / r_{1}=0.4, \sigma_{z} / r_{1}=0.3$ and $e=0.2$. The deconvolution of the mean velocity field (Fig. 6b), obtained from the statistical simulation, is performed with the four methods presented above. Comparing the mean velocity field corrected for the wandering effects (Fig. 12) with the actual velocity field, shown in Fig. 6a, it is evident that all four methods perform a sufficiently adequate deconvolution, even if the Van Cittert algorithm generates a certain error in proximity of the boundary of the space domain, due to the finiteness of the latter.
Fig. 12 Deconvolution of the mean cross-velocity field with different methods. Wandering simulation with $\sigma_{y} / r_{1}=0.4$, $\sigma_{z} / r_{1}=0.3, e=0.2$ : a Van Cittert algorithm; b RichardsonLucy algorithm; c blind algorithm; d direct deconvolution in the Fourier domain. (Colour scale: white $=0$ and black $=0.4$ )
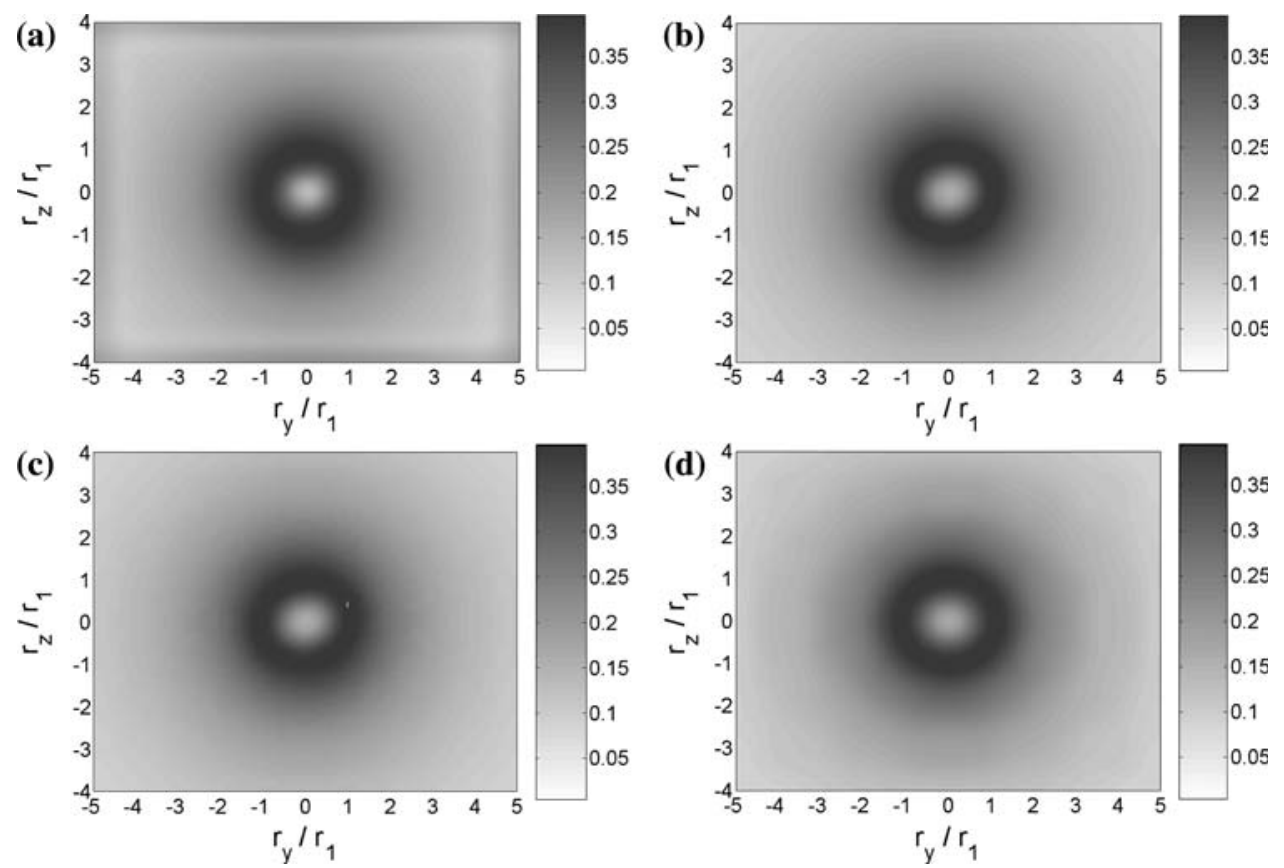
Fig. 13 Deconvolution of the mean cross-velocity field with different methods. Wandering simulation with $\sigma_{y} / r_{1}=0.4$, $\sigma_{z} / r_{1}=0.3, e=0.2$ : a traverse through the mean vortex center along the spanwise direction; b traverse through the mean vortex center along the normal direction

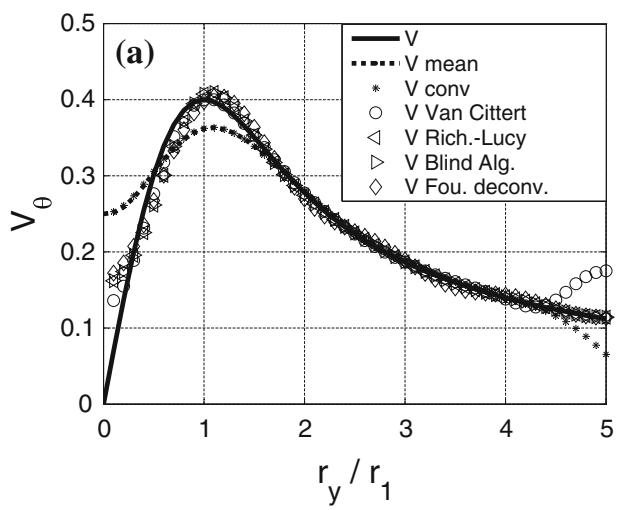

In Fig. 13 the sections through the mean vortex center, along both the spanwise and the normal directions, of the cross-velocity field are reported. As can be seen, the deconvolution of the mean field performed by all four methods allows the peak cross-velocity and the core radius to be evaluated very accurately. However, the error is still large at the mean vortex center, where the cross-velocity should be zero, by assuming the radial velocity to be negligible. Furthermore, the error produced by the Van Cittert algorithm near to the space domain boundary is evident.

Two test cases with extreme conditions are now presented. In the first case the anisotropy parameter, $e$, was set equal to 1 . Figure 14 shows that an adequate deconvolution of the mean cross-velocity field is achieved but a residual anisotropy is still present, with the exception of the direct deconvolution performed in the Fourier domain.

In the second case the wandering amplitude in the spanwise direction is set equal to the core radius; as expected, the prediction of the actual velocity field is very inaccurate (Fig. 15). For the spanwise direction, with the greater wandering amplitude, the best correction is performed by the direct deconvolution in the Fourier domain, whereas for the normal direction this method is the worst. Moreover, the boundary error produced by the Van Cittert algorithm becomes significant even though the space domain is large with respect to the core radius.
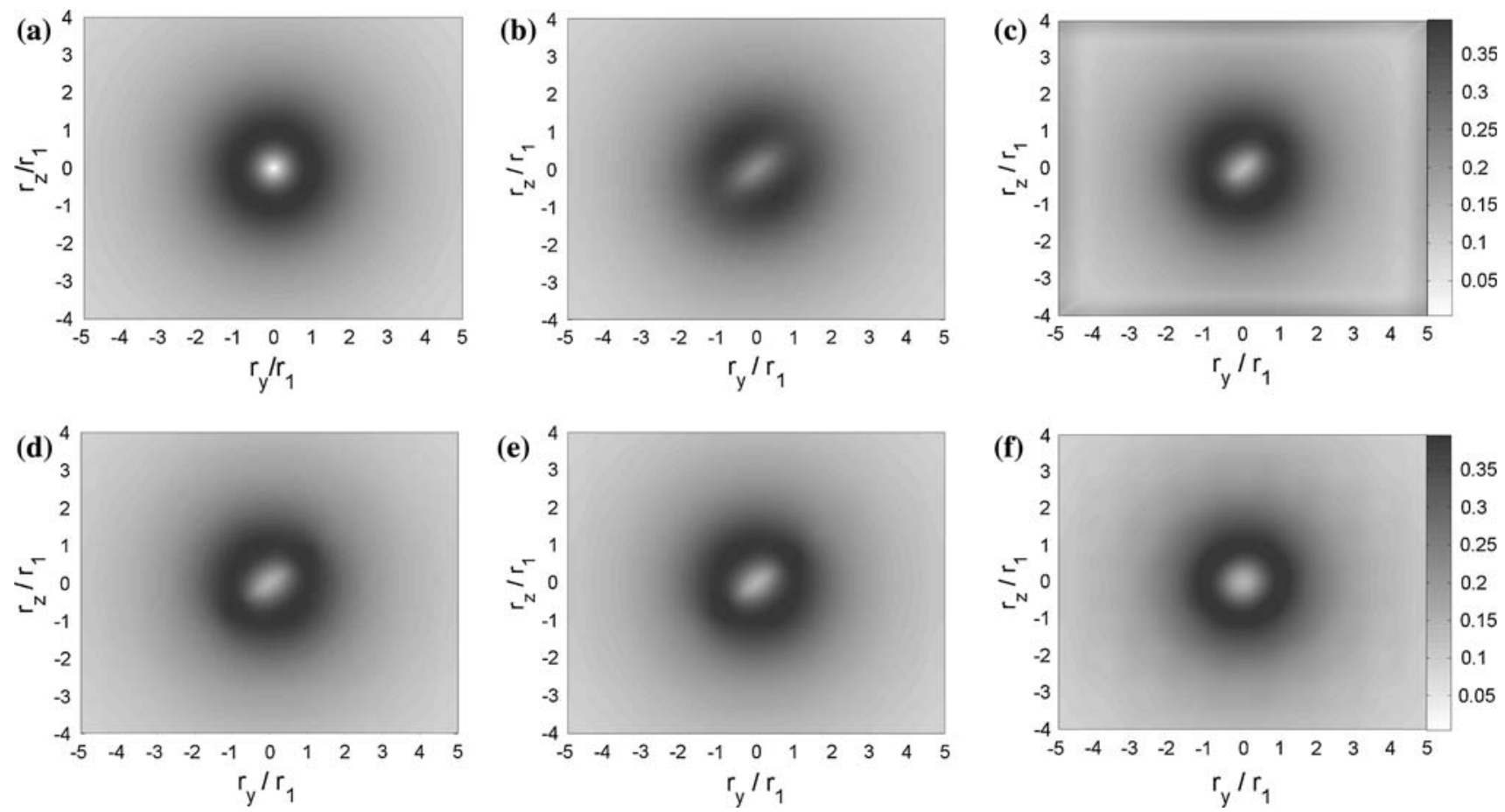

Fig. 14 Wandering simulation with $\sigma_{\gamma} / r_{1}=0.4, \sigma_{z} / r_{1}=0.3, e=1$ : a actual cross-velocity field; $\mathbf{b}$ mean cross-velocity field; c deconvolution with the Van Cittert algorithm; $\mathbf{d}$ deconvolution with Richardson-Lucy algorithm; $\mathbf{e}$ deconvolution with the blind algorithm; $\mathbf{f}$ deconvolution with the direct deconvolution in the Fourier domain. (Colour scale: white $=0$ and black $=0.4$ ) 
Fig. 15 Deconvolution of the mean cross-velocity field with different methods. Wandering simulation with $\sigma_{y} / r_{1}=1$, $\sigma_{z} / r_{1}=0.3, e=0$ : a traverse through the mean vortex center along the spanwise direction; b traverse through the mean vortex center along the normal direction

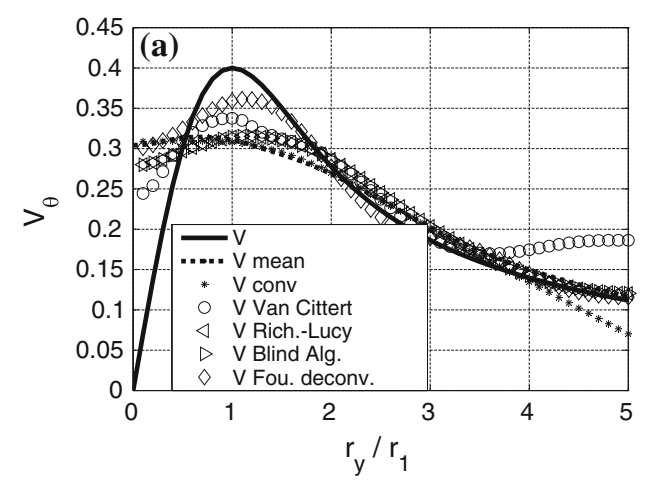

The typical percent error encountered in the prediction of the peak cross-velocity is reported in Fig. 16 as a function of the actual wandering amplitude, evaluated as the average of at least three different values of the normal wandering amplitude and of the anisotropy parameter. Errors on the correction of $V_{\theta 1}$ are very limited for wandering amplitudes smaller than $60 \%$ of the core radius, while for larger amplitudes the errors increase, even if they remain less than $10 \%$ of the actual value.

In conclusion, for limited wandering amplitudes the corrections performed by all four methods are able to effectively remove the wandering smoothing effects from the mean velocity fields and to accurately predict the actual core radius and the actual peak cross-velocity of the vortices. However, the Van Cittert algorithm presents an error at the space domain boundary that can become significant when this boundary is too close to the locations of interest, and for all methods a repeatable error occurs at the mean vortex center. Nonetheless, it should be pointed out that these residual errors at the vortex center do not invalidate the procedure for the evaluation of the wandering amplitude. In effect, as described in Sect. 3 and shown in Fig. 7, a satisfactory assessment of this quantity may be obtained from the RMS values and the gradients of the velocity components obtained from the original data, i.e., those affected by the wandering effects.

For very anisotropic wandering, a residual anisotropy on the corrected actual velocity field was found for all methods except for the direct deconvolution in the Fourier domain. Finally, for wandering amplitudes comparable to the core radius, the correction is not very accurate, and in this case the statistical simulations show that even the wandering amplitudes are poorly evaluated from static measurements.

\section{Correction of the static measurements for wandering smoothing effects}

The whole procedure to evaluate the wandering characteristics from static measurements and to correct the mean

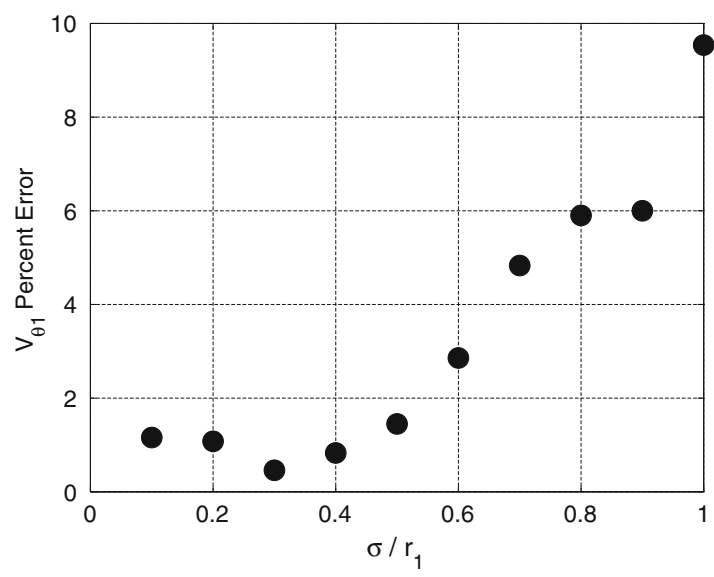

Fig. 16 Error on the deconvolution of the peak cross-velocity, $V_{\theta 1}$, as percentage of the actual value, as a function of the wandering amplitude

velocity field for wandering effects was applied to the static measurements obtained experimentally with the 5HP, already presented in Sect. 2.

Regarding the measurement grids, only the data upstream of the location $x / c=1.5$ were analysed, as the remaining measurement grids were too coarse for this analysis. The condition $U_{\infty}=10 \mathrm{~m} / \mathrm{s}, \alpha=8^{\circ}, x / c=0.33$ is first presented. The RMS values of $V$ and $W$ at the mean vortex center location, needed to calculate the wandering amplitudes, were evaluated from the traverse data, as the longer sampling time $(33 \mathrm{~s})$ and the higher sampling rate $(2 \mathrm{kHz})$, with respect to the grids data, allow a more accurate evaluation of those parameters to be achieved. In addition, the prediction of the wandering amplitudes requires the slope of the tangential velocity at the mean vortex center, which is generally calculated from the mean circulation, obtained from the traverse data, fitted through Hoffmann \& Joubert's method. For the condition being considered the wandering amplitude in the spanwise direction is $\sigma_{y} / c=4.225 \times 10^{-3}$ and in the normal one it is slightly smaller $\sigma_{z} / c=3.826 \times 10^{-3}$, corresponding to $\sigma_{y} / r_{1}=0.125$ and $\sigma_{z} / r_{1}=0.114$, respectively. It is evident 
that wandering is not very significant for the present streamwise location. Furthermore, for the analysed condition the anisotropy parameter was found to be $e=0.031$.

The covariance matrix $\Sigma$ corresponding to the $2 \mathrm{VdF}$, which represents the wandering, is:

$\Sigma=\left(\begin{array}{cc}\sigma_{y}^{2} & e \sigma_{y} \sigma_{z} \\ e \sigma_{y} \sigma_{z} & \sigma_{z}^{2}\end{array}\right)$

The direction of the principal axes of the wandering may be evaluated from the eigenvectors of the covariance matrix, $\Sigma$, which is also used to evaluate the wandering amplitudes along the principal axes from the square root of its eigenvalues. For the present condition the principal axes are rotated by $8.69^{\circ}$, measured from the spanwise direction to the normal one. This shows that the largest wandering amplitude, $\sigma_{1} / r_{1}=0.126$, is along the upward-outboard to downward-inboard direction, while the orthogonal amplitude is $\sigma_{2} / r_{1}=0.113$.

The measured mean cross-velocity field, i.e., the modulus of the velocity component lying on a plane orthogonal to the vortex axis, was corrected to remove the wandering smoothing effects with the four methods presented in Sect. 4. The sections of the cross-velocity field in the spanwise and normal directions through the mean vortex center, plotted in Fig. 17, show that all methods perform a comparable correction and that the effects of wandering on the mean velocity field are, as expected, almost negligible for the present location $x / c=0.33$.

The correction of the measurements carried out at the location $x / c=1.5$ is now presented. The measured mean non-dimensional cross-velocity field is plotted in Fig. 18a, but restricted to the space domain used for the wandering correction. All maps of velocity fields corresponding to this location are plotted with a grey scale where the white colour indicates the zero value and the black the value 0.6. For this condition the wandering amplitude in the spanwise direction is $\sigma_{y} / r_{1}=0.416$ and in the normal direction $\sigma_{z} / r_{1}=0.338$. The anisotropy parameter was found to be $e=0.344$. Consequently, the principal axes of wandering are rotated with respect to the frame of reference by $29.4^{\circ}$, measured from the spanwise to the normal direction, and the wandering amplitudes along these peculiar directions are $\sigma_{1} / r_{1}=0.447$ and $\sigma_{2} / r_{1}=0.295$. The consequent $2 V d F$, which represents the wandering at this location, is shown in Fig. 18b.

The correction of the wandering effects on the mean non-dimensional cross-velocity field was then performed with the four correction methods. As can be seen in Fig. 19, the correction performed with the Van Cittert algorithm is completely inadequate because the boundary of the space domain is too close to the vortex core for the observed wandering amplitudes. Consequently, the
Fig. 17 Correction of the measured mean nondimensional cross-velocity field from wandering effects for the condition $\alpha=8^{\circ}$,

$U_{\infty}=10 \mathrm{~m} / \mathrm{s}, x / c=0.33$ : a spanwise section through the mean vortex center; b normal section through the mean vortex center
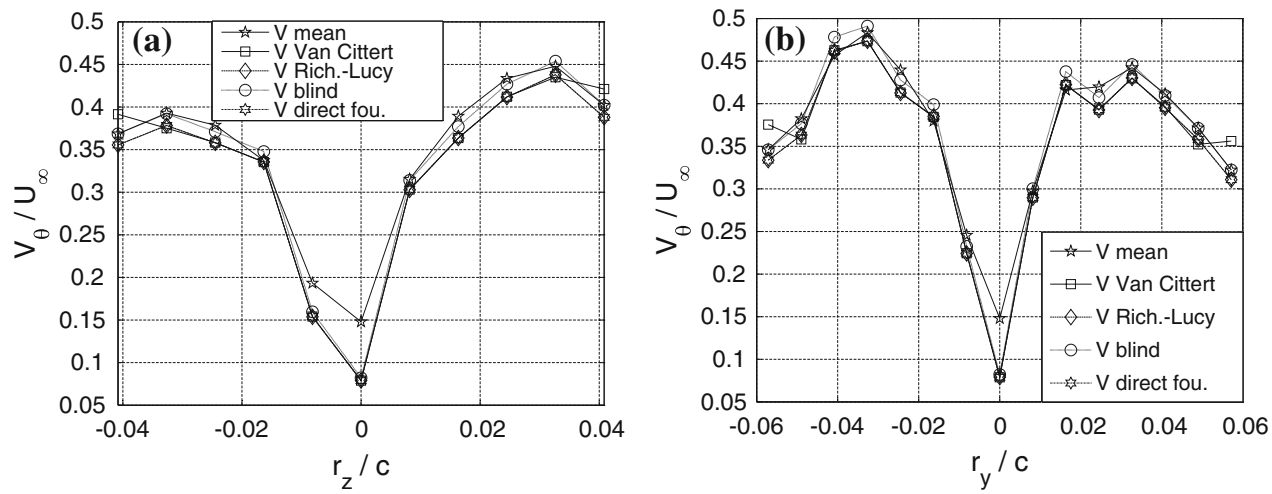

Fig. 18 Correction of the static measurements carried out with the 5HP for the condition $\alpha=8^{\circ}, U_{\infty}=10 \mathrm{~m} / \mathrm{s}$, $x / c=1.5$ : a mean nondimensional cross-velocity field (colour scale: white $=0$ and black $=0.6$ ); $\mathbf{b}$ bi-variate normal probability density function, $2 V d F$, which represents the wandering (colour scale: white $=0$ and black $=0.06$ )
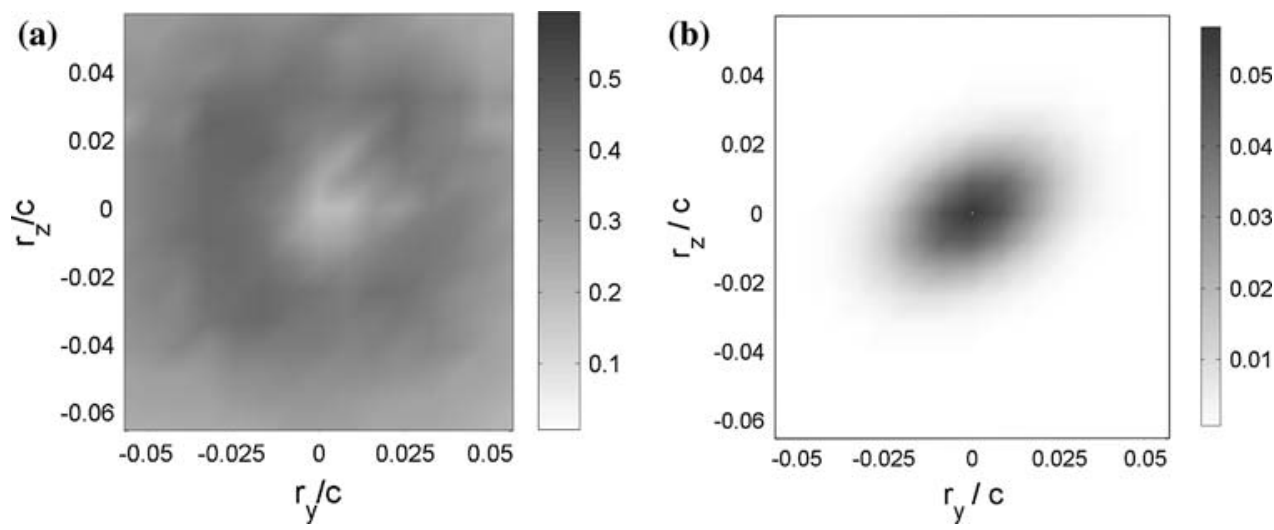
Fig. 19 Correction of the mean non-dimensional cross-velocity field obtained from the 5HP static measurements for the condition $U_{\infty}=10 \mathrm{~m} / \mathrm{s}$, $\alpha=8^{\circ}, x / c=1.5:$ a Van Cittert algorithm; b Richardson-Lucy algorithm; c blind algorithm; d direct deconvolution in the Fourier domain. (Colour scale: white $=0$ and black $=0.6$ )
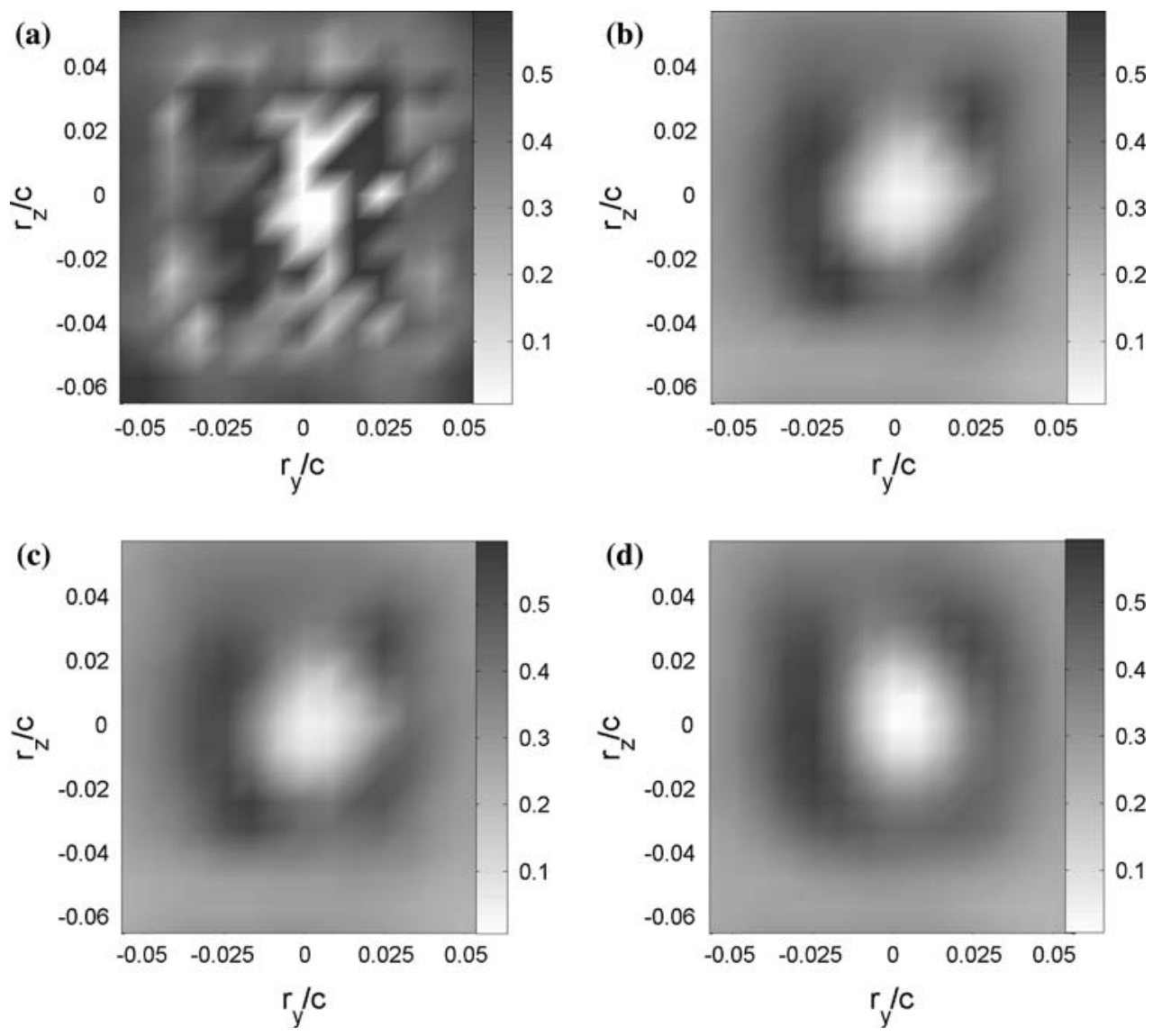

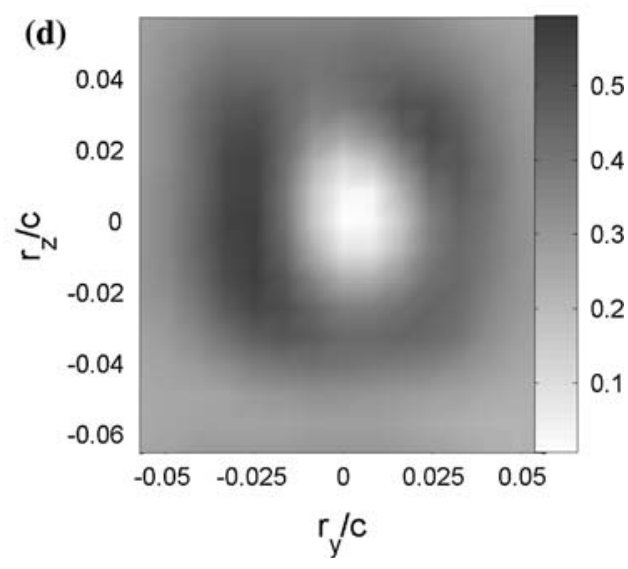

boundary error typical of this method has a large influence on the prediction of the actual velocity field.

In Fig. 20 sections of the velocity fields through the mean vortex center highlight that the corrections performed by all the methods, excluding the Van Cittert algorithm (not reported), are comparable and, as expected, greater in the spanwise direction than in the normal one, as the wandering is more intense in the spanwise direction. Moreover, the non-dimensional peak cross-velocity varies from the measured value of about $V_{\theta 1} / U_{\infty}=0.44$ to the corrected value $V_{\theta 1} / U_{\infty}=0.57$.
The wandering characteristics for all conditions and locations, evaluated from the traverse data, are reported in Table 2 . The wandering amplitude is generally larger in the spanwise direction than along the normal direction. Considering the tests performed by varying the streamwise distance from the wing, it is seen that the wandering amplitudes grow by proceeding downstream, as found by Devenport et al. (1996). The wandering amplitudes vary from almost $13 \%$ of the core radius at $x / c=0.33$ up to $90 \%$ for $x / c=3$.

As for the tests performed with different free-stream velocities, at variance with the findings of Devenport et al.
Fig. 20 Correction of the measured cross-velocity field relative to the condition $U_{\infty}=10 \mathrm{~m} / \mathrm{s}, \alpha=8^{\circ}$, $x / c=1.5:$ a section through the mean vortex center along the spanwise direction; b section through the mean vortex center along the normal direction
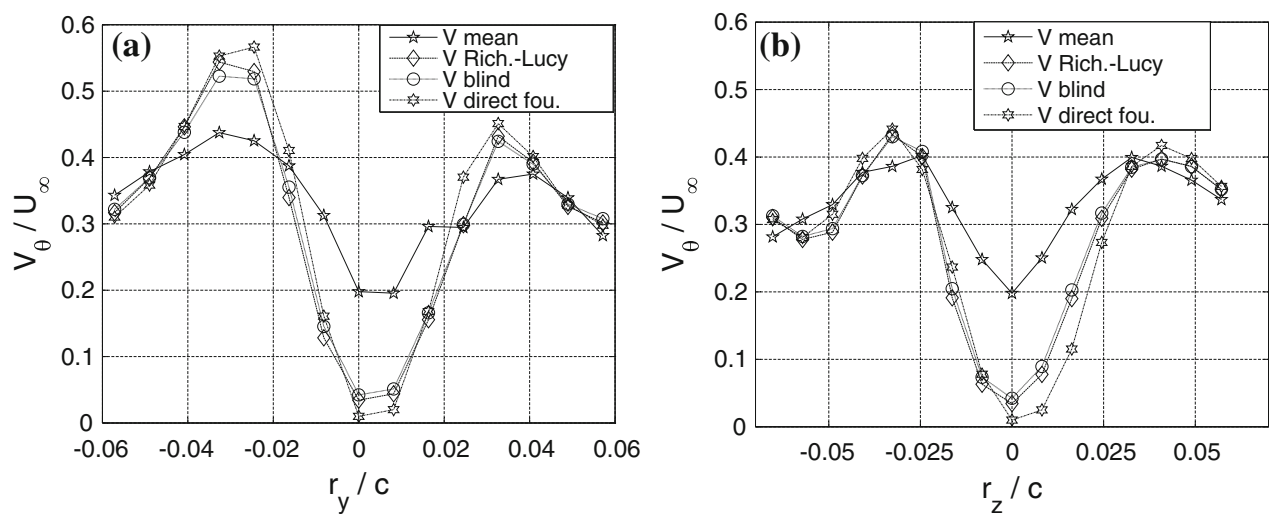
Table 2 Wandering parameters for the tested conditions and locations

\begin{tabular}{lllllllllll}
\hline$N_{o}$ & $\alpha(\mathrm{deg})$ & $U_{\infty}(\mathrm{m} / \mathrm{s})$ & $x / c$ & $\sigma_{y} / c$ & $\sigma_{z} / c$ & $\sigma_{y} / r_{1}$ & $\sigma_{z} / r_{1}$ & $\sigma_{1} / r_{1}$ & $\sigma_{2} / r_{1}$ & $\Theta(\mathrm{deg})$ \\
\hline G 01 & 8 & 10 & 0.1 & 0.004 & 0.004 & 0.149 & 0.151 & 0.16 & 0.14 & -48.6 \\
G 02 & 8 & 10 & 0.33 & 0.004 & 0.004 & 0.125 & 0.114 & 0.126 & 0.113 \\
G 03 & 8 & 10 & 0.66 & 0.007 & 0.006 & 0.224 & 0.174 & 0.233 & 0.163 & 21.8 \\
G 04 & 8 & 10 & 1 & 0.012 & 0.01 & 0.33 & 0.279 & 0.345 & 0.26 & 26.2 \\
G 05 & 8 & 10 & 1.5 & 0.016 & 0.013 & 0.416 & 0.338 & 0.447 & 0.295 & 29.4 \\
G 06 & 8 & 10 & 2 & 0.016 & 0.012 & 0.489 & 0.368 & 0.543 & 0.282 & 30.6 \\
G 07 & 8 & 10 & 3 & 0.036 & 0.026 & 0.849 & 0.621 & 0.925 & 0.501 & 28.2 \\
G 08 & 8 & 10 & 6 & 0.068 & 0.062 & 1.26 & 1.15 & 1.45 & 0.888 & 39.5 \\
G 09 & 8 & 20 & 6 & 0.012 & 0.011 & 0.291 & 0.261 & 0.33 & 0.209 \\
G 10 & 8 & 30 & 6 & 0.005 & 0.004 & 0.154 & 0.122 & 0.159 & 0.116 \\
G 11 & 4 & 10 & 6 & 0.091 & 0.077 & 1.25 & 1.07 & 1.39 & 0.885 \\
G 12 & 12 & 10 & 6 & 0.028 & 0.022 & 0.565 & 0.447 & 0.632 & 0.345 \\
\hline
\end{tabular}

(1996) the wandering amplitudes were found to be dramatically reduced with the increase of the free-stream velocity. Furthermore, the tests carried out with different angles of attack showed that the wandering amplitudes, $\sigma_{y} / c$ and $\sigma_{z} / c$, decrease almost linearly with increasing angle of attack, contrary to the findings of Rokhsaz et al. (2000). All these results suggest that wandering decreases as the strength of the vortex increases, as already observed by Devenport et al. (1996).

The orientation of the principal axes of wandering $\Theta$, measured from the spanwise to the normal direction, was evaluated from the eigenvectors of the covariance matrix generated by $\sigma_{y}, \sigma_{z}$ and $e$. The $\Theta$ values in Table 2 show that wandering occurs predominantly in the upward-outboard to downward-inboard direction, except for the location $x / c=0.1$. $\Theta$ increases by proceeding in the downstream direction and almost reaches an asymptotic value, whereas it is difficult to detect a clear trend of $\Theta$ with varying angle of attack or free-stream velocity because only three different values of these parameters were tested.

Summarizing, the wandering amplitudes, $\sigma_{y} / c$ and $\sigma_{z} / c$, grow by proceeding downstream, and decrease with increasing angle of attack and free-stream velocity. Consequently, even if it is difficult to draw firm conclusions about the origin of wandering, from the present tests we can exclude that wandering be a self-induced phenomenon, as had been proposed by Rokhsaz et al. (2000). In effect, wandering is always reduced with increasing strength and level of concentration of the vortices, i.e., when the vortices have more energy to oppose external disturbances.

Finally, the correction of the data obtained from the traverses is presented. However, it is first appropriate to recall that wandering is considered to be a $2 \mathrm{D}$ motion which occurs predominantly orthogonally to the vortex axis, and that in this work it has been characterized by a bi-variate normal probability density function, $2 V d F$, as proposed by Devenport et al.
(1996). Now, the $2 V d F$ probability density function can be evaluated as the product of $1 \mathrm{D}$ probability density functions along the principal axes of wandering, because the probability density functions along these two peculiar directions are statistically independent. Consequently, the principal axes of wandering are the only directions where it is possible to perform a proper $1 \mathrm{D}$ deconvolution of the mean velocity field, taking a 2D motion into account.

This suggests that, considering the long testing times required for performing closely-spaced measurement grids, an easier procedure may be envisaged to characterize a wing-tip vortex. In particular, preliminary and sufficiently accurate measurements may be carry out around the mean vortex center, whose location can easily be determined. This would allow $\sigma_{y}, \sigma_{z}$ and $e$ to be calculated, and the principal axes of wandering and relative wandering amplitudes to be estimated. Subsequently, fine traverses can be performed along the principal axes and the data may be corrected for wandering effects using 1D deconvolutions.

However, the present traverse measurements were carried out along the spanwise and the normal directions, so that the $1 \mathrm{D}$ deconvolution of the mean velocity field with the section of the $2 \mathrm{VdF}$ along the considered direction does not produce the actual velocity field. For this reason the correction of the traverse data, measured in the spanwise direction, was performed through the $1 \mathrm{D}$ deconvolution of the mean velocity field with two 1D normal probability density functions: the first with wandering amplitude $\sigma_{1}$, the larger wandering amplitude along the principal axes, and the second with wandering amplitude equal to $\sigma_{2}$. The former produces the greatest correction connected to wandering, the latter produces the smallest one. Therefore, this technique provides a confidence interval of the actual velocity field that may be obtained from the 1D measurements.

Examples of the obtained corrections of the traverse data from wandering effects is shown in Fig. 21. It is evident 
Fig. 21 Correction of the crossvelocity obtained from traverses performed through the mean vortex center in the spanwise direction: a $U_{\infty}=10 \mathrm{~m} / \mathrm{s}$, $\alpha=8^{\circ}, x / c=1$;

b $U_{\infty}=10 \mathrm{~m} / \mathrm{s}, \alpha=8^{\circ}$, $x / c=3$

Table 3 Correction of the peak cross-velocity, $V_{\theta 1}$, from wandering effects along the principal axes of wandering
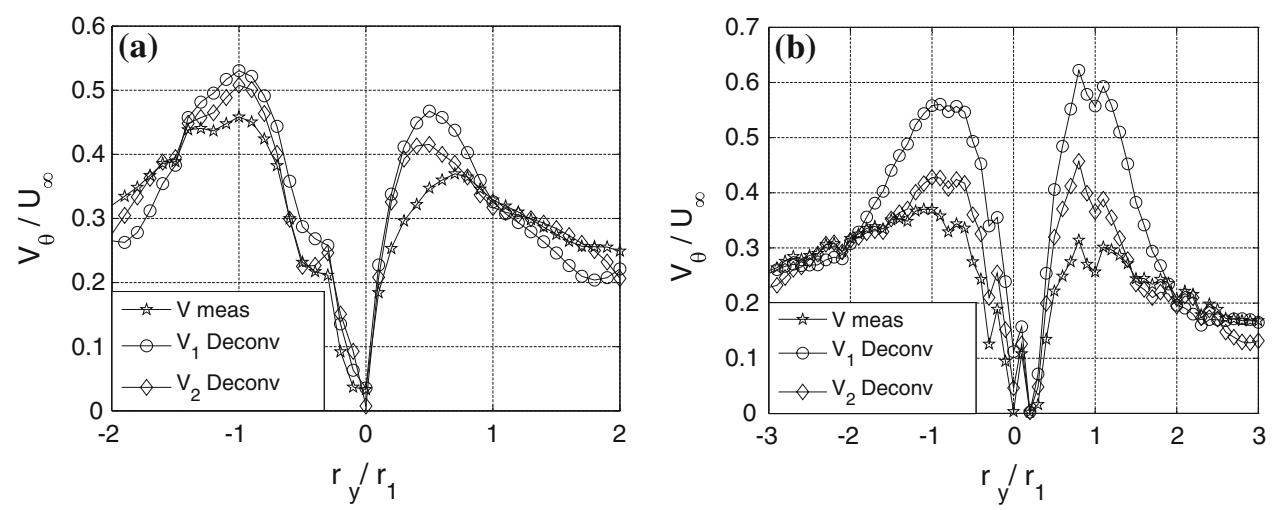

\begin{tabular}{lllllll}
\hline$N_{o}$ & $\alpha(\mathrm{deg})$ & $U_{\infty}(\mathrm{m} / \mathrm{s})$ & $x / c$ & $V_{\theta 1} / U_{\infty}$ & $\begin{array}{l}V_{\theta 1} / U_{\infty} \text { corr. } \\
\text { with } \sigma_{1}\end{array}$ & $\begin{array}{l}V_{\theta 1} / U_{\infty} \text { corr. } \\
\text { with } \sigma_{2}\end{array}$ \\
\hline G 01 & 8 & 10 & 0.1 & 0.58 & 0.619 & 0.606 \\
G 02 & 8 & 10 & 0.33 & 0.576 & 0.588 & 0.586 \\
G 03 & 8 & 10 & 0.66 & 0.569 & 0.604 & 0.603 \\
G 04 & 8 & 10 & 1 & 0.395 & 0.476 & 0.452 \\
G 05 & 8 & 10 & 1.5 & 0.356 & 0.435 & 0.395 \\
G 06 & 8 & 10 & 2 & 0.346 & 0.468 & 0.383 \\
G 07 & 8 & 10 & 3 & 0.284 & 0.529 & 0.363 \\
G 08 & 8 & 10 & 6 & 0.182 & 0.529 & 0.389 \\
G 09 & 8 & 20 & 6 & 0.316 & 0.362 & 0.337 \\
G 10 & 8 & 30 & 6 & 0.358 & 0.382 & 0.359 \\
G 11 & 4 & 10 & 6 & 0.098 & 0.249 & 0.181 \\
G 12 & 12 & 10 & 6 & 0.297 & 0.375 & 0.313 \\
\hline
\end{tabular}

that with this technique a range for the predicted vortex core velocity field is obtained, even if the actual one is not exactly evaluated. For the location $x / c=1$ wandering amplitudes are roughly $30 \%$ of the core radius and the maximum correction of the peak cross-velocity is about $20 \%$ of the measured value, but for the location $x / c=3$ a significant correction of about $86 \%$ of the measured value is performed; however, the results of the statistical simulations suggest that the latter value is probably not very accurate, as the wandering amplitudes are comparable to the core radius.

Finally, the measured peak cross-velocities are compared with the corresponding corrected values in Table 3 for all conditions and locations. The trend of the peak cross-velocity corrected for wandering effects is generally unchanged, suggesting that the vortex is effectively diffusing due to viscosity by proceeding downstream, but at a reduced rate with respect to the measured values.

\section{Conclusions}

The aims of the present work were to evaluate the possibility of characterizing vortex wandering using static measurements and to compare different methods to correct the measured velocity fields for wandering smoothing effects. Statistical simulations of the wandering of a LambOseen vortex have been performed. The wandering locations have been represented through a bi-variate normal probability density function and the vortex center location of each snapshot has been generated using the statistical software $R$. The statistical simulations highlighted that wandering amplitudes smaller than $60 \%$ of the core radius are well predicted from the ratio between the RMS value of the cross-velocity and its slope measured at the mean vortex center. With increasing wandering amplitudes the predictions become more inaccurate, showing errors up to $35 \%$ of the actual value for wandering amplitudes comparable to the core radius. Moreover, it was found that the anisotropy parameter, and thus the principal axes, can be determined from the opposite of the value of the crosscorrelation coefficient between the spanwise and the normal velocities measured at the mean vortex center.

The correction of the wandering smoothing effects on the mean velocity field has then been executed through the deconvolution of the latter with the bi-variate normal probability density function representing wandering. Four different algorithms were used, which avoid any 
assumption or any fitting of the measured velocity field: the Van Cittert algorithm, the Richardson-Lucy algorithm, the blind deconvolution and the numerical direct deconvolution in the Fourier domain.

The results show that the correction methods allow wandering smoothing effects to be removed from the mean velocity fields, even if an error remains at the mean vortex center. In other words, the actual core radius and the actual peak cross-velocity (which are the most interesting quantities, providing the size and intensity of the vortex) can be accurately predicted, but the corrected velocity fields can only be considered as artificial ones, due to an apparent violation of the conservation equations. Nevertheless, it was shown that this error near the vortex center has no consequence for the evaluation of the wandering amplitudes, which require only quantities derived from the original data, i.e., those affected by the wandering effects.

More in detail, the Van Cittert algorithm suffers from a repeatable error in correspondence to the space domain boundary, which can be significant when this boundary is very close to the locations of interest. For highly anisotropic wandering the best correction is achieved through the numerical direct deconvolution in the Fourier domain. However, this correction is not very accurate for wandering amplitudes comparable to the core radius. In any case, the statistical simulations show that in these conditions even the wandering amplitudes are inadequately predicted from static measurements.

Static measurements of a vortex generated from a NACA 0012 half-wing model have been carried out with a fast-response five hole pressure probe. The mean velocity field was corrected for wandering smoothing effects with the four methods that were previously assessed through the statistical simulations. It was found that, in extreme circumstances, the peak cross-velocity corrected for wandering effects was $70 \%$ larger than the measured value.

The experimental measurements have shown vortex wandering to be predominantly in the upward-outboard to downward-inboard direction. Furthermore, the wandering amplitudes grow by proceeding downstream, whereas they decrease by increasing either the wing angle of attack or the free-stream velocity. In general, wandering is reduced when the vortex strength increases or for more concentrated vortices, so the conclusion may by drawn that probably it is not a self-induced phenomenon.

Acknowledgments The authors would like to thank M. Morelli, who made a useful contribution to the planning of the tests at CSIR, and G. Lombardi for many useful discussions. Thanks are also due to F. Flandoli for his essential suggestions on statistics. Finally, the authors are grateful to G. Barbaro for his contribution in the execution of the tests.

\section{References}

Bandyopadhyay P, Stead D, Ash R (1991) Organized nature of a turbulent trailing vortex. AIAA J 29:1627-1633

Batchelor G (1964) Axial flow in trailing line vortices. J Fluid Mech 20:645-658

Beninati M, Marshall J (2005) An experimental study of the effect of free-stream turbulence on a trailing vortex. Exp Fluids 38:244257

Chigier N, Corsiglia V (1972) Wind-tunnel studies of wing wake turbulence. J Aircraft 9:820-825

Chow J, Zilliac G, Bradshaw P (1997) Mean and turbulence measurements in the near field of a wingtip vortex. AIAA J 35:1561-1567

Corsiglia V, Schwind R, Chigier N (1973) Rapid scanning, threedimensional hot-wire anemometer surveys of wing-tip vortices. J Aircraft 12:752-757

Devenport W, Rife M, Liapis S, Follin G (1996) The structure and development of a wing-tip vortex. J Fluid Mech 326:67-106

Gerner A, Maurar C (1981) Calibration of seven-hole probes suitable for high angles in subsonic compressible flow. Tech. rep., USAFA-TR-81-4

Green S, Acosta A (1991) Unsteady flow in trailing vortices. J Fluid Mech 227:107-134

Gursul I, Xie W (2000) Origin of vortex wandering over delta wings. J Aircraft 37:348-350

Heyes A, Hubbard S, Marquis A, Smith D (2003) On the roll-up of a trailing vortex sheet in the very near field. Proc Inst Mech Eng G J Aerospace Eng 217:217-269

Heyes A, Jones R, Smith D (2004) Wandering of wing-tip vortices. In: 12th International symposium on application of laser techniques to fluid mechanics, Lisbon, Portugal

Hoffmann E, Joubert P (1963) Turbulent line vortices. J Fluid Mech 16:395-411

Iungo G, Skinner P (2007) Correction of wandering effects on static measurements of a wing tip vortex. Tech. Rep. ADIA 2007-2, Atti del Dipartimento di Ingegneria Aerospaziale dell'Università di Pisa

Jansson PA (1984) Deconvolution with application in spectroscopy. Academic Press, New York

Jaquin J, Fabre D, Geffroy P, Coustols E (2001) The properties of a transport aircraft wake in extended near field: an experimental study. AIAA Paper 1038

Larson J (2002) Two-dimensional and three-dimensional blind deconvolution of fluorescence confocal images. In: Proceedings of SPIE

Richardson WH (1972) Bayesian-based iterative method of image restoration. J Opt Soc Am 62(55):820-825

Ripley B (1987) Stochastic Simulation. ISBN 047181884 4. Wiley, New York

Rokhsaz K, Foster S, Miller L (2000) Exploratory study of aircraft wake vortex filaments in a water tunnel. J Aircraft 37:1022-1027

Shekarriz A, Fu T, Katz J, Liu H, Huang T (1992) Study of junction and tip vortices using particle displacement velocimetry. AIAA J 30:145-152

Yeung A, Lee B (1999) Particle image velocimetry study of wing-tip vortices. J Aircraft 36:482-484 